\title{
SRF expedites metastasis and modulates the epithelial to mesenchymal transition by regulating miR-199a-5p expression in human gastric cancer
}

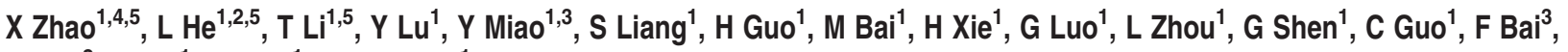

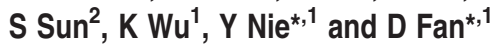

Dysregulation of transcription factors (TFs) is associated with tumor progression, but little is known about TF expression patterns in the context of gastric cancer (GC) metastasis. Using array-based profile analysis, we found that 22 TFs showed differential activities between GC cell lines with low- and high-metastatic potential. Of this group of TFs, serum response factor (SRF) was significantly upregulated in metastatic GC cells. SRF expression was frequently elevated in a panel of metastatic GC cells and tissues, and high-level expression of SRF was significantly associated with a more aggressive phenotype and poor prognosis in patients with GC. In GC cell lines, overexpression of SRF potently promoted cell migration and invasion in vitro as well as the formation of intrahepatic and pulmonary metastases in vivo, whereas loss of SRF inhibited GC cell invasion and metastasis. We also performed a microRNA microarray to screen for transcriptional targets of SRF and found that SRF transactivates miR-199a-5p and miR-199a-3p by directly binding to their promoters. We further determined that overexpression of miR-199a-5p, but not miR-199a-3p, increased GC cell invasion and metastasis. In contrast, inhibition of miR-199a-5p impaired the metastatic potential of GC cells in vitro and in vivo, and E-cadherin was identified as a direct and functional target of miR199a-5p in GC cells. Specifically, our results showed that SRF promotes GC metastasis and the epithelial to mesenchymal transition (EMT) though miR-199a-5p-mediated downregulation of E-cadherin. The present study thus provides insight into the specific biological behavior of SRF in GC metastasis. As increased activity of the SRF/miR-199a-5p/E-cadherin pathway appears to promote GC cell EMT and metastasis, these regulators may therefore be developed as therapeutic targets or biomarkers for GC progression.

Cell Death and Differentiation (2014) 21, 1900-1913; doi:10.1038/cdd.2014.109; published online 1 August 2014

Gastric cancer (GC) is the fourth most common cancer and the second leading cause of cancer-related death worldwide. ${ }^{1}$ The major cause of death and relapse from GC is metastasis, which greatly hampers the success of treatment modalities. ${ }^{2,3}$ The process of metastasis consists of multiple sequential steps, including the escape of tumor cells into surrounding tissues, followed by their intravasation into vessels, survival in the circulatory system, extravasation, and growth into macroscopic secondary tumors in distant organs. ${ }^{4}$ The initial metastatic cascade stage depends on the prominent biological event known as the epithelial to mesenchymal transition (EMT), which is characterized by specific morphogenetic changes, cell-cell adhesion loss, and increased cell motility. ${ }^{5}$ Many studies have demonstrated that aberrant expression of certain transcription factors (TFs), including serum response factor (SRF), AP1, and NF- $\kappa \mathrm{B}$, can contribute to metastasis in various cancer types. ${ }^{6-8}$ However, the TF expression patterns in the context of GC metastasis have not been fully explored.

SRF is a MCM1, Agamous, Deficiens, and SRF family TF involved in several cellular activities, including proliferation, differentiation, angiogenesis, and apoptosis. ${ }^{9,10}$ Recently, it was proposed that SRF acts as a pro-metastatic factor during tumor metastasis. Treisman and colleagues ${ }^{6}$ demonstrated that SRF depletion in breast cancer cells could reduce cell invasion and motility in culture and their ability to colonize the lung. SRF's role in gastrointestinal (Gl) cancers has received particular attention. Moon et al. reported that SRF is upregulated in hepatocellular carcinoma (HCC) and colorectal carcinoma (CRC), and ectopic SRF expression was shown to enhance their metastatic potential. ${ }^{11-14}$ However, the potential function and underlying mechanisms of SRF in GC metastasis have not been well defined.

\footnotetext{
${ }^{1}$ State Key Laboratory of Cancer Biology \& Institute of Digestive Diseases, Xijing Hospital of Digestive Diseases, the Fourth Military Medical University, Xi'an, Shaanxi, China; ${ }^{2}$ Department of Nephrology, Xijing Hospital, the Fourth Military Medical University, Xi'an, Shaanxi, China and ${ }^{3}$ Department of Gastroenterology, the People's Hospital of Ningxia Hui Nationality Autonomous Region, Yinchuan, Ningxia Hui Autonomous Region, China

${ }^{*}$ Corresponding author: Dr Y Nie or Dr D Fan, State Key Laboratory of Cancer Biology, Fourth Military Medical University, Xijing Hospital of Digestive Diseases, 127 Chang Le West Road, Xi'an 710032, Shaanxi, China. Tel: +86 29 84771506; Fax: +86 29 82539041; E-mail: yongznie@fmmu.edu.cn or daimingfan @fmmu.edu.cn ${ }^{4}$ Present address: Stem Cell Program, Children's Hospital, Harvard Medical School, Boston, MA 02115, USA.

${ }^{5}$ These authors contributed equally to this work.

Abbreviations: AP1, activator protein 1; ChIP, chromatin immunoprecipitation; CRC, colorectal cancer; CREB, CAMP response element-binding protein; EMT, epithelial to mesenchymal transition; GAPDH, glyceraldehyde 3-phosphate dehydrogenase; GC, gastric cancer; HCC, hepatocellular carcinoma; IHC, immunohistochemistry; LMB, leptomycin B; MADS, MCM1, Agamous, Deficiens, and SRF; miRNA, microRNA; NF- $\kappa B$, nuclear factor kappa-light-chain-enhancer of activated B cells; PCR, polymerase chain reaction; siRNA, short interfering RNA; shRNA, short hairpin RNA; snRNA, small nuclear RNA; SRF, serum response factor; TF, transcription factor; UTR, untranslated region

Received 09.12.13; revised 30.6.14; accepted 02.7.14; Edited by R De Maria; published online 01.8.14
} 
MicroRNAs (miRNAs) are endogenous 18-24 nucleotide single-stranded RNA molecules that act as posttranscriptional gene expression regulators. In particular, they interact with the $3^{\prime}$-untranslated region $\left(3^{\prime}\right.$-UTR) of target mRNAs to inhibit protein translation or promote mRNA degradation. ${ }^{15}$ Many studies have documented the role of miRNAs in processes required for metastasis, including cell migration, invasion, and $E M T,{ }^{16}$ and a recent study suggests that miRNAs are tightly linked to TFs in gene regulatory networks. ${ }^{17}$ Several studies have shown that SRF can directly regulate miRNAs in the context of smooth muscle cell proliferation and differentiation, ${ }^{18,19}$ adding further complexity to SRF regulatory activities. However, it remains largely unknown whether SRF regulates miRNAs during tumor metastasis.

Here, we show that SRF is significantly upregulated in metastatic GC cells and promotes GC cell migration, invasion, and metastasis both in vitro and in vivo. Moreover, we provide evidence that SRF coordinates gene expression networks involved in metastasis through miRNA-mediated mechanisms and, more specifically, that SRF may modulate GC metastasis through miR-199a-5p transactivation. Further, we identified E-cadherin as a direct and functional miR-199a-5p target. Importantly, we found that the SRF/miR-199a-5p/E-cadherin pathway leads to both inhibition of E-cadherin and SRFinduced EMT. Collectively, our results provide a novel explanation for GC metastasis and link its aggressive nature to the interaction between SRF, miR-199a-5p, and the tumor suppressor E-cadherin.

\section{Results}

Screening of metastasis-related transcriptional participants by high-throughput TF activity profiling. To identify TFs that drive GC metastasis, we compared TF activity profiles between parental GC9811 cells and their metastatic derivative, GC9811-P cells. Array-based DNA-binding TF activity profiles revealed that 17 TFs were elevated and 5 were downregulated in GC9811-P cells relative to GC9811 cells (Supplementary Table S1). Two distinctive TF expression clusters were obtained by hierarchical clustering analysis (Figure 1a). The upregulated TFs included AP1, $\mathrm{NF}-\kappa \mathrm{B}$, and $\mathrm{CREB}$, which have been reported to be elevated in several cancers and to promote invasion and/or metastasis. ${ }^{7,8,20}$ Among these TFs, SRF has been reported in the regulation of genes encoding actin cytoskeleton and contractile proteins. ${ }^{21}$ Because the cytoskeleton controls cell motility, invasion, and adhesion, we further focused on determining whether SRF has a pivotal role in GC metastasis. We first investigated the relationship between endogenous SRF levels and GC metastatic potential. Western blot analysis showed that endogenous SRF was elevated in highly metastatic GC9811-P and MKN28-M cells compared with their low metastatic counterparts, GC9811 and MKN28-NM cells (Figure 1b). Real-time PCR analysis revealed that SRF was significantly upregulated in lymph node metastases compared with matched primary GC tissues and adjacent non-tumor gastric tissues (Figure 1c). These findings indicate that SRF may affect GC metastasis.

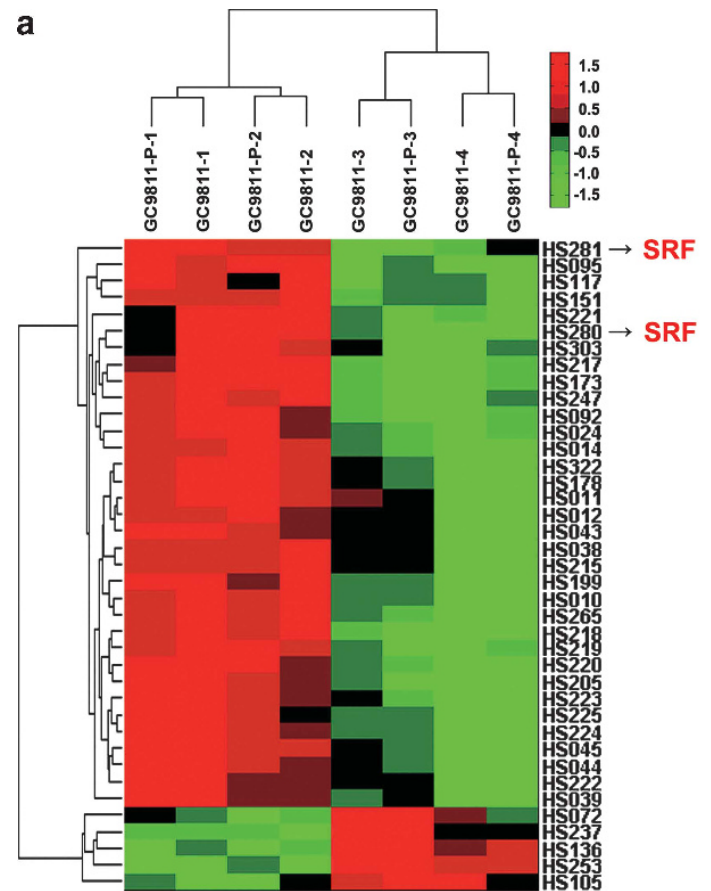

b
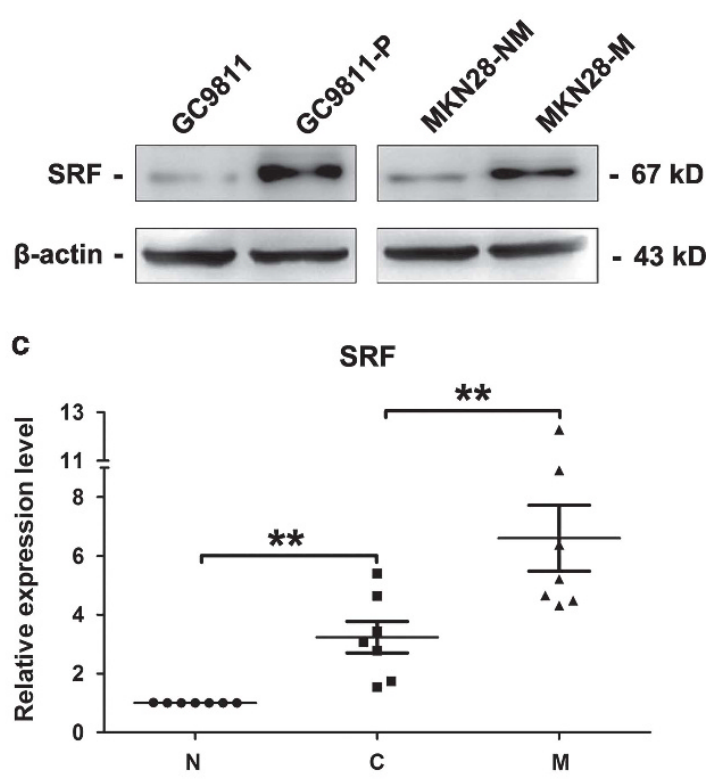

Figure 1 Systematic identification and expression evaluation of GC metastasis-related TFs. (a) Hierarchical clustering of TFs activated or repressed ( $q<0.056$, fold $>1.5$ ) in GC9811 and GC9811-P cells. The scale bar depicts the S.D. from the mean. (b) western blot analysis of SRF protein levels in GC9811, GC9811-P, MKN28-NM, and MKN28-M cells. $\beta$-actin was used as a loading control. (c) Real-time PCR analysis of endogenous SRF levels in seven groups of primary GC tissues (C), matched adjacent non-tumor gastric tissues (N), and lymph node metastatic tissues (M). Real-time PCR values were normalized to U6 small nuclear RNA (snRNA) levels, and all experiments were performed in triplicate. ${ }^{*} P$-value $<0.05 ;{ }^{* *} P$-value $<0.01$ 
SRF overexpression and nuclear translocation occur in metastatic cells and tissues and are correlated with GC progression. We further investigated the clinicopathological and prognostic significance of SRF in GC patients. By immunohistochemistry, we found that SRF was significantly upregulated in metastatic GC tissues compared with primary GC tissues and non-tumor gastric tissues (Figure 2a, Supplementary Table S2). We then assessed SRF levels in a cohort of $106 \mathrm{GC}$ tissues. Correlation analysis showed that high-level SRF expression in GC tissues was significantly associated with a more aggressive tumor phenotype $(P<0.05$, Table 1). Kaplan-Meier analysis further revealed that high-level SRF expression was associated with shorter disease-free survival in GC patients $(P<0.01$, Figure $2 \mathrm{~b}$, Supplementary Table S3). Additional multivariate Cox regression analyses indicated that high-level SRF expression served as an independent prognostic factor for poor survival in GC patients ( $P<0.01$, Supplementary Table S3).

Nuclear translocation of TFs controls their transcriptional regulatory activity. ${ }^{22}$ We found that SRF was predominantly located in the cytoplasm of GC9811 cells, whereas it was primarily localized to the nuclei of GC9811-P cells (Figures 2c and d). In GC patient tissues, SRF expression was generally increased in the nuclei but drastically decreased in the
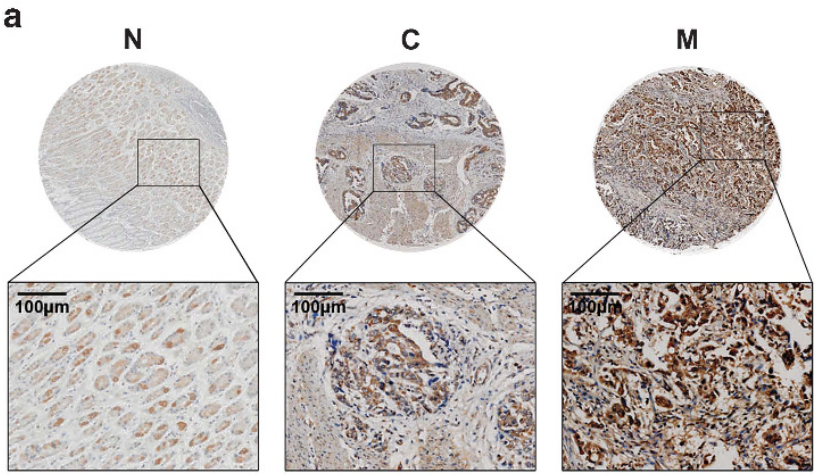

b

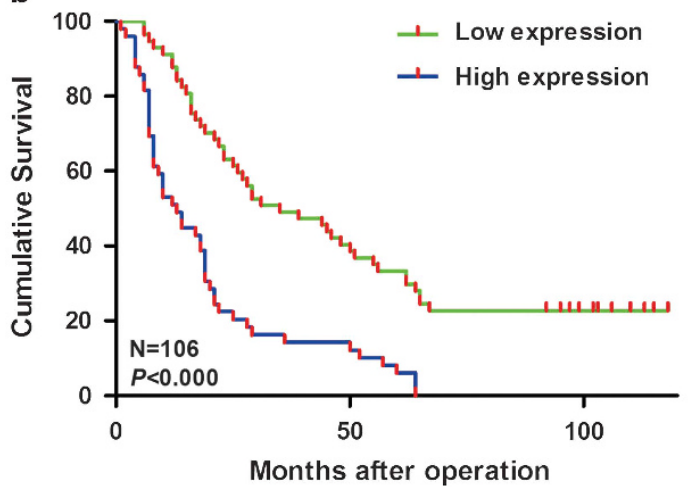

c

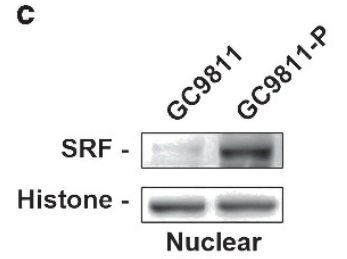

e

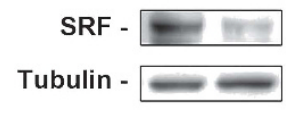

Cytoplasmic

\section{(1)}

SRF -

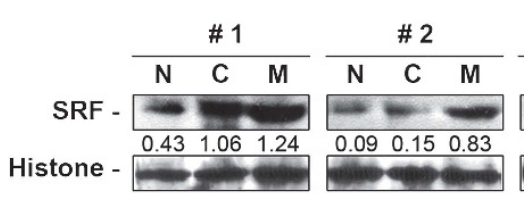

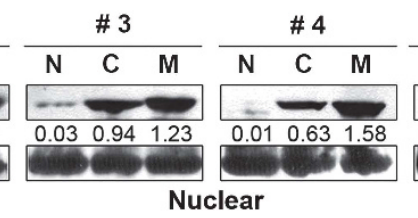

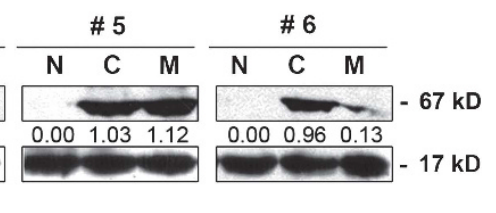
\begin{tabular}{c} 
SRF - \\
Tubulin - $1.09 \quad 1.12 \quad 0.25$ \\
\hline
\end{tabular}

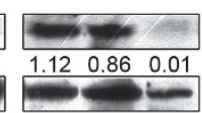

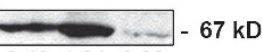

$\begin{array}{lll}0.46 & 1.24 & 0.02\end{array}$ $\begin{array}{rrrr}0.46 & 1.24 & 0.02 \\ -50 & 60\end{array}$

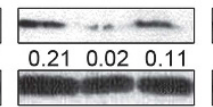

Cytoplasmic d
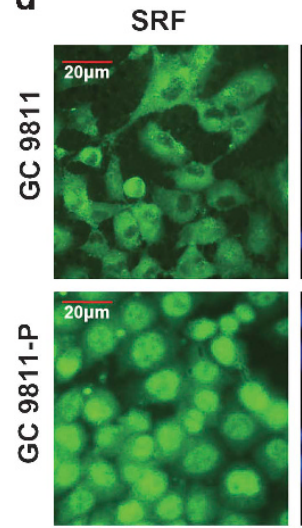

DAPI
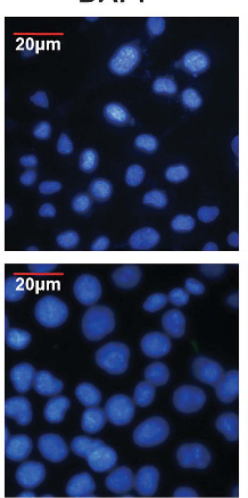

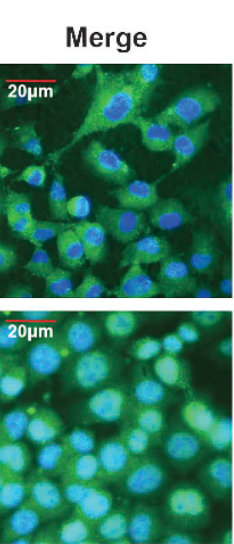

f

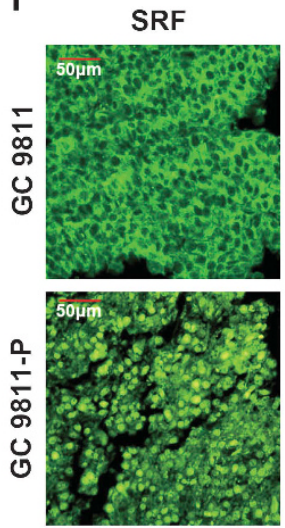

DAPI
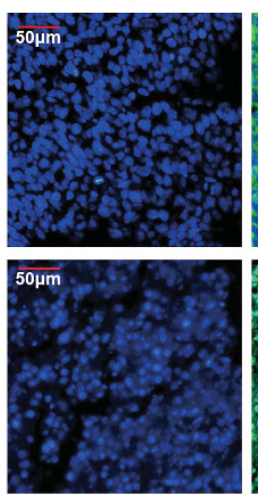

Figure 2 Validation of SRF expression and its subcellular localization in GC cells and tissues. (a) Representative immunostained specimens showing SRF expression in normal gastric tissues ( $N ; n=25)$, primary $\mathrm{GC}$ tissues $(\mathrm{C} ; n=28)$, and metastatic $\mathrm{GC}$ tissues $(\mathrm{M} ; n=48)$. (b) Kaplan-Meier analysis of overall survival according to high or low SRF expression. (c) Western blot analysis of nuclear and cytoplasmic SRF levels in GC9811 and GC9811-P cells, and (d) in six groups of GC samples. Histone and tubulin were used as loading controls for nuclear and cytoplasmic extracts, respectively. (e) Immunofluorescence staining to assess subcellular SRF localization in GC9811 and GC9811-P cells, and (f) tumor samples from recipient mice 
Table 1 Correlation of SRF expression in tissue with patients' clinicopathological variables in 106 cases of GC

\begin{tabular}{|c|c|c|c|c|}
\hline \multirow[t]{2}{*}{ Variables } & \multicolumn{3}{|c|}{ Expression of SRF } & \multirow[t]{2}{*}{$P$-value ${ }^{a}$} \\
\hline & $\begin{array}{c}\text { All cases } \\
n=106\end{array}$ & $\begin{array}{c}\text { Low } \\
\text { expression } \\
n=57\end{array}$ & $\begin{array}{c}\text { High } \\
\text { expression } \\
n=49\end{array}$ & \\
\hline Gender & & & & 0.840 \\
\hline Male & 67 & 37 & 30 & \\
\hline Female & 39 & 20 & 19 & \\
\hline Age (years) & & & & 0.697 \\
\hline$\leqslant 57.1$ & 55 & 31 & 24 & \\
\hline$>57.1$ & 51 & 26 & 25 & \\
\hline Tumor size $(\mathrm{cm})$ & & & & 0.564 \\
\hline$\leqslant 5$ & 51 & 29 & 22 & \\
\hline$>5$ & 55 & 28 & 27 & \\
\hline Grade of differentiation & & & & 0.000 \\
\hline G1 & 6 & 5 & 1 & \\
\hline $\mathrm{G} 2$ & 46 & 34 & 12 & \\
\hline G3 & 54 & 18 & 36 & \\
\hline Depth of invasion ${ }^{\mathrm{b}}$ & & & & 0.000 \\
\hline $\mathrm{T} 1$ & 10 & 10 & 0 & \\
\hline T2 & 9 & 9 & 0 & \\
\hline T3 & 79 & 35 & 44 & \\
\hline $\mathrm{T} 4 \mathrm{a} / \mathrm{b}$ & 2 & 0 & 2 & \\
\hline Lymph node status & & & & 0.002 \\
\hline NO & 21 & 18 & 3 & \\
\hline N1 & 11 & 8 & 3 & \\
\hline $\mathrm{N} 2$ & 27 & 12 & 15 & \\
\hline $\mathrm{N} 3 \mathrm{a} / \mathrm{b}$ & 47 & 19 & 28 & \\
\hline Distant metastasis & & & & 0.036 \\
\hline MO & 99 & 56 & 43 & \\
\hline M1 & 7 & 1 & 6 & \\
\hline
\end{tabular}

Mean age at operation

a $\chi^{2}$-test

${ }^{b}$ Data of six cases are missing

cytoplasm of lymph node metastatic tissues (Figure 2e). These different subcellular SRF distribution patterns were also observed in vivo in transplanted tumors originating from GC9811 or GC9811-P cell subcutaneous injections into nude mice (Figure 2f). Together, these results suggest that SRF upregulation and nuclear translocation frequently occur during GC metastasis and may therefore have a vital role in this process.

SRF affects GC cell adhesion, invasion, and metastasis in vitro and in vivo. To determine whether SRF regulates GC cell invasion and metastasis, we constructed lentiviral SRF (LV-SRF) and SRF shRNA (LV-shSRF) vectors and assayed the in vitro migration and invasion abilities of transduced cells. SRF inhibition led to a significant migration and invasion reduction compared with control cells (Figure 3a). By contrast, SRF-expressing GC9811 cells exhibited a remarkable increase in cell migration and invasion potential (Figure 3b). We then examined whether SRF regulates cell-matrix adhesion, an essential process for metastasis initiation and metastatic cell homing to tissues. ${ }^{23}$ Interestingly, SRF inhibition enhanced cell adhesion (Figure 3c), whereas SRF overexpression suppressed cell adhesion (Figure 3d). To elucidate SRF's function in vivo, GC9811-P cells infected with LV-shSRF were delivered into nude mice by tail vein injection, and the number of intrahepatic and pulmonary metastatic nodules was clearly decreased in the SRF-silenced group (Figure $3 e$ ). In contrast, we observed the opposite results when mice were injected with GC9811 cells infected with LV-SRF (Figure 3f). Similar results were obtained when using MKN28-NM and MKN28-M cells (Supplementary Figure S2). Together, these findings indicate that SRF has an important role in regulating GC cell metastasis both in vitro and in vivo.

SRF transcriptionally regulates miR-199a-5p and $-3 p$ expression by directly targeting the miR-199a-1 and -2 promoters. SRF target genes are characterized by the presence of the SRF-binding consensus element, the CArG box. ${ }^{10}$ Recent studies estimated that one-third or more of mammalian miRNA genes contain at least one promoter region CArG box. ${ }^{18}$ We thus speculated that certain miRNAs might be SRF targets involved in GC metastasis. Using miRNA microarrays, we identified 84 miRNAs that were differentially expressed when SRF was silenced in GC9811-P cells (Figure 4a). Among them, miR-199a-5p and miR-199a-3p, both of which are processed from primary miR-199a (pri-miR-199a) and contain a CArG box in their promoter regions, were downregulated when SRF was knocked down. Real-time PCR analysis further confirmed that endogenous miR-199a-5p and $-3 p$ were increased upon SRF upregulation in GC9811-P cells (Figure 4b) and reduced upon SRF inhibition (Figure 4c), indicating that SRF may contribute to miR-199a-5p and $-3 p$ upregulation.

Pri-miR-199a is transcribed from two loci located on chromosomes 19 (miR-199a-1) and 1 (miR-199a-2). To determine whether pri-miR-199a is transcriptionally activated by SRF, we constructed luciferase reporter plasmids containing a wild-type or mutant SRF-binding site upstream of the miR-199a-1 and -2 coding sequences (Figure 4d). Luciferase reporter assays revealed that the wild-type miR-199a-1 and -2 promoters showed statistically significant activity following SRF expression in GC9811-P cells; however, luciferase activity was decreased when the mutant constructs were tested. In contrast, this transcriptional activation effect was not evident in GC9811 cells. Importantly, ectopic overexpressing SRF in GC9811 cells caused an increase of luciferase activity in wild-type miR-199a-1 and -2 promoters, recapitulating the effect of transcriptional activation observed in GC9811-P cells (Figure 4e). In addition, chromatin immunoprecipitation (ChIP) analyses revealed that SRF could specifically bind to CArG elements in the miR-199a-1 and -2 promoters in GC9811-P cells (Figure 4f), providing strong evidence that SRF can directly regulate miR-199a-5p and $-3 p$.

To test whether SRF's subcellular distribution affects miR-199a-3p and $-5 p$ expression, we treated GC cells with leptomycin B (LMB), a nuclear export inhibitor, to block the translocation of SRF. Inhibiting the nuclear export of SRF produced no significant changes in miR-199a-5p and $-3 p$ expression in GC9811-P cells but increased their expression 
a

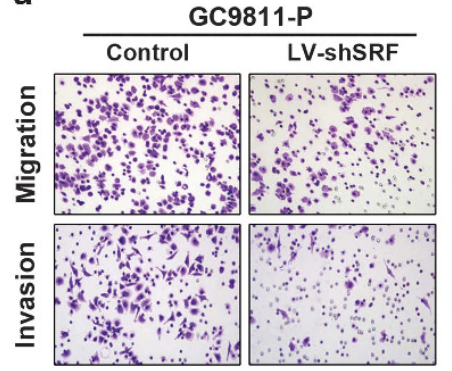

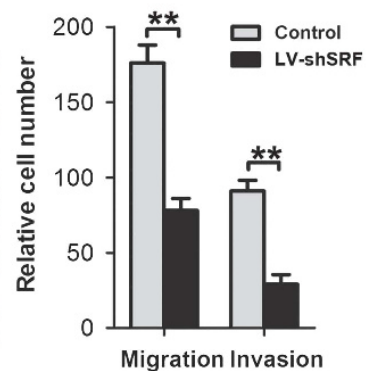

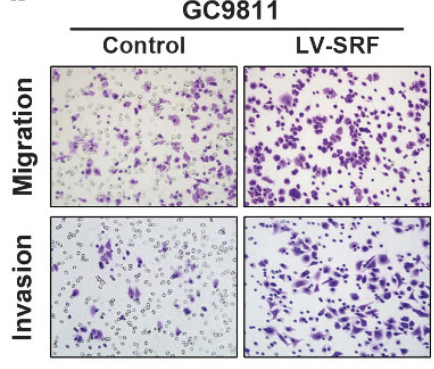

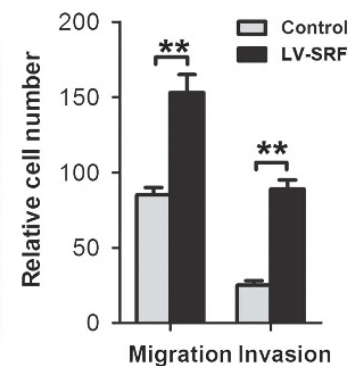

C

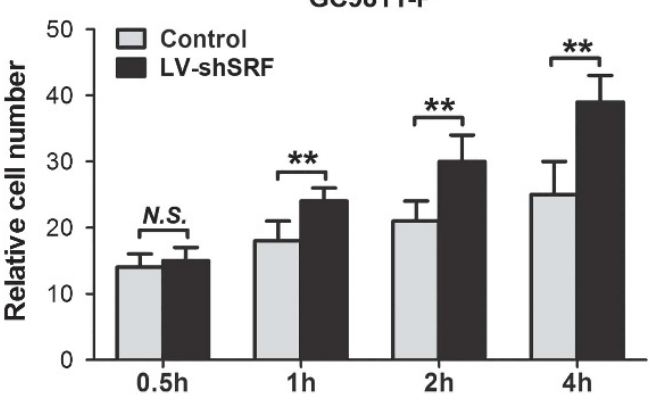

d

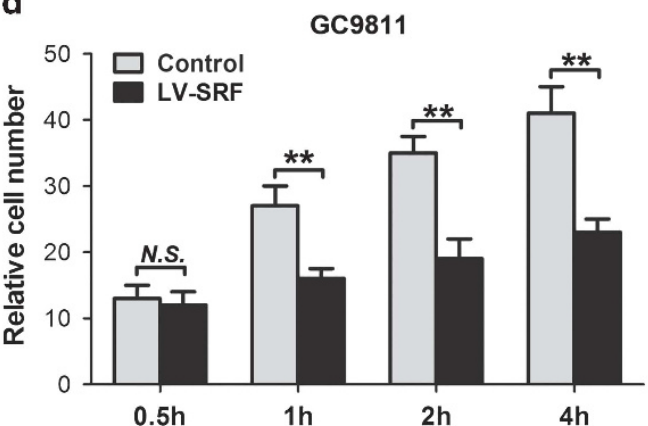

e
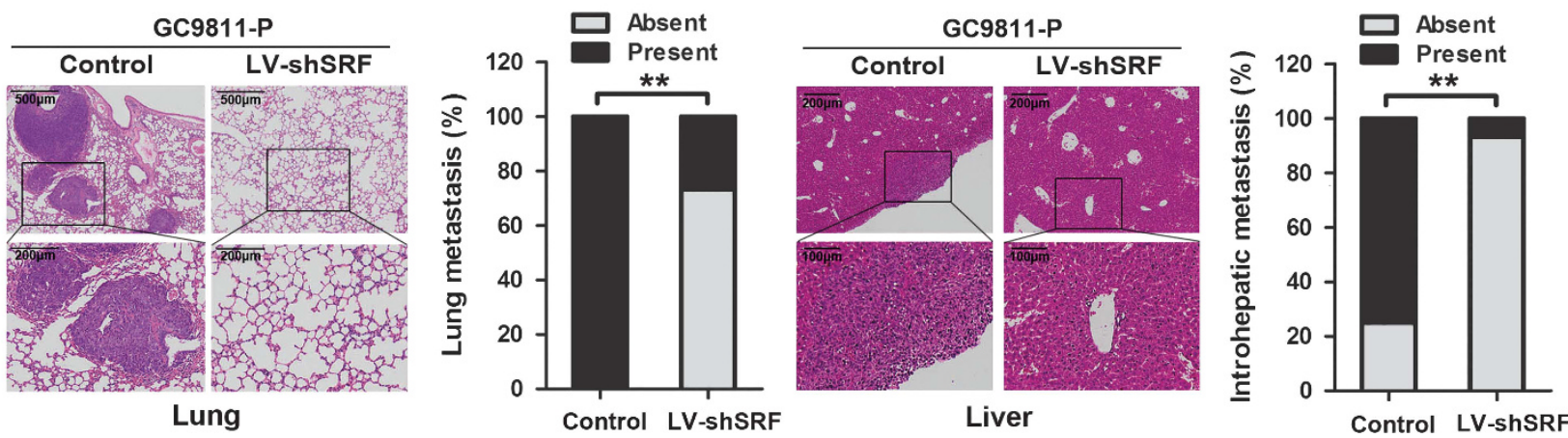

f

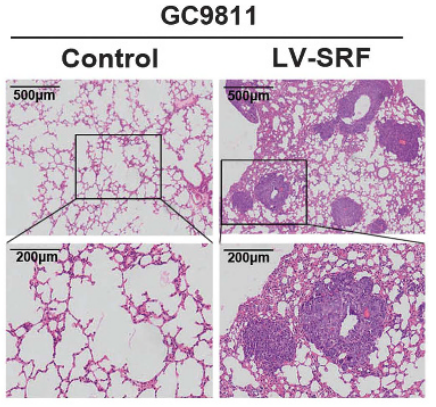

Lung

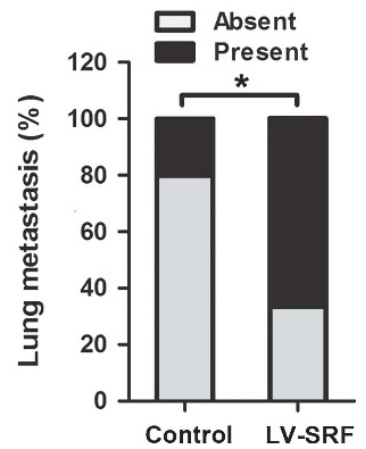

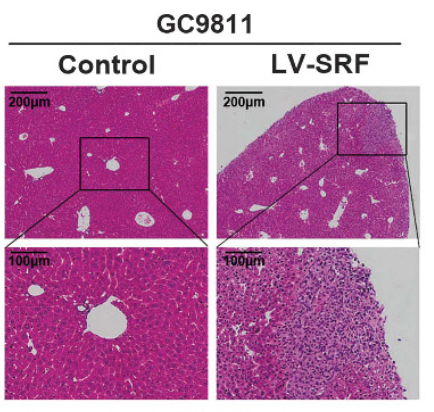

Liver

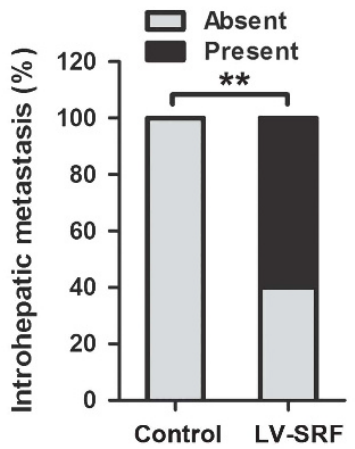

Figure 3 SRF regulates GC cell adhesion, migration, invasion and metastasis in vitro and in vivo. (a) Transwell migration and invasion assays using GC9811-P cells infected with LV-shSRF or negative control (control) and (b) GC9811 cells infected with LV-SRF or control. Representative images are shown on the left, and quantification of 10 randomly selected fields is shown on the right. Magnification $=\times 200$. (c) Cell adhesion assays. Bar graphs represent the average numbers of adhesive cells on the Matrigel after $0.5,1,2$, and $4 \mathrm{~h}$ for GC9811-P cells infected with the LV-shSRF or control, and (d) GC9811 cells infected with the LV-SRF or control. (e) Representative hematoxylin and eosin (H\&E)-stained intrahepatic and pulmonary metastases of GC9811-P cells infected with LV-shSRF or control, and (f) GC9811 cells infected with the LV-SRF or control. Bar graphs represent the percentage of mice with or without metastatic nodules in the liver or the lungs. ${ }^{*} P$-value $<0.05$; ${ }^{*} P$-value $<0.01$; NS, not significant

in GC9811 cells (Figure $4 \mathrm{~g}$ and Supplementary Figure S3), suggesting that SRF displacement may be sufficient to induce target gene transcription.
Ectopic expression of miR-199a-5p, but not miR-199a-3p, regulates GC cell adhesion, invasion, and metastasis. We measured endogenous miR-199a-5p and $-3 p$ expression 
levels in the GC samples described above and found that tissues from lymph node metastases expressed higher miR-199a-5p and $-3 p$ levels compared with primary GC tissues and adjacent normal gastric tissues. Furthermore, statistical analysis revealed that miR-199a-5p and $-3 p$ expression levels positively correlated with SRF expression (Figures $5 a$ and b), indicating that miR-199a-5p and $-3 p$ expression is correlated with SRF expression and GC progression.

We next evaluated whether miR-199a mediates the SRFinduced promotion of invasion and metastasis in GC cells. In vitro migration and invasion assays revealed that miR199a-5p overexpression promoted GC cell migration and invasion (Figure 5c). In contrast, miR-199a-5p inhibition led to a significant decrease in GC9811-P cell in migration and invasion (Figure 5d). Of note, neither miR-199a-3p overexpression nor inhibition significantly affected these processes. Adhesion assays showed that miR-199a-5p overexpression suppressed GC9811 cell adhesion (Figure 5e), whereas miR-199a-5p inhibition enhanced GC9811-P cell adhesion (Figure 5f). Moreover, an in vivo metastasis assay showed that infected with lentiviral miR199a-5p inhibitor resulted in a significant decrease in the number of intrahepatic and pulmonary metastatic nodules in GC9811 cells (Figure 5g). Similar results were obtained when using MKN28-NM and MKN28-M cells (Supplementary Figure S4). Together, these results indicate that miR-199a-5p can promote GC cell invasion and metastasis and that miR-199a-5p inactivation can abolish the enhanced metastatic potential of GC cells.

E-cadherin is a direct and functional miR-199a-5p target in GC cells. To understand the underlying mechanism by which miR-199a-5p promotes GC invasion and metastasis, we used several computational methods to identify potential targets. Among these targets, $\mathrm{CDH} 1$, which encodes E-cadherin, was of particular interest because its expression has been found to be progressively lost in several cancer types, and it is involved in the suppression of migration and invasion. $^{24}$ To determine whether $\mathrm{CDH} 1$ is a direct miR199a-5p target, we constructed reporter constructs using wild-type or mutant CDH1 $3^{\prime}$-UTR fragments (Figure 6a). Luciferase reporter assays revealed that miR-199a-5p overexpression in GC9811 cells suppressed the wild-type $\mathrm{CDH} 1$ $3^{\prime}$-UTR reporter but did not affect the mutant CDH1 $3^{\prime}$-UTR luciferase reporter (Figure $6 \mathrm{~b}$ ). In addition, real-time PCR assays showed that neither overexpression nor inhibition of miR-199a-5p altered CDH1 mRNA levels (Figure 6c). However, by Western blot analysis, we observed that miR-199a-5p overexpression in GC9811 cells significantly suppressed E-cadherin protein levels, whereas miR-199a-5p inhibition in GC9811-P cells increased E-cadherin expression (Figure 6d), indicating that miR-199a-5p regulates $\mathrm{CDH} 1$ at the posttranscriptional level. To ascertain whether decreased E-cadherin levels might account for the increase in cell migration and invasion observed following miR-199a$5 p$ overexpression, we transfected GC9811 cells with miR$199 a-5 p$ and then with an E-cadherin construct or control vector. In vitro migration and invasion assays demonstrated that constitutive E-cadherin expression significantly reduced the miR-199a-5p-induced increase in GC cell migration and invasion (Figure 6e). Collectively, these results suggest that $\mathrm{E}$-cadherin is both a direct and functional miR-199a-5p target.

\section{Endogenous SRF downregulates E-cadherin by trans-} activating miR-199a-5p in metastatic GC cells. The inverse relation between SRF and E-cadherin has been observed in Gl cancers, including HCC and CRC, and we confirmed that E-cadherin protein levels were significantly reduced upon SRF overexpression in GC9811 cells and increased upon SRF silencing in GC9811-P cells (Figure 6f). However, the exact mechanisms underlying this SRFinduced E-cadherin repression remained unclear. We first speculated that the decrease in E-cadherin levels depended on SRF transcriptional repression. Although our bioinformatic analysis revealed an SRF-binding site in the $\mathrm{CDH} 1$ promoter, subsequent ChIP assays demonstrated that SRF could not directly bind to the $\mathrm{CDH} 1$ promoter; thus, SRF does not directly regulate E-cadherin (Figure $6 \mathrm{~g}$ ). We then tested the possibility that the SRF-induced miR-199a-5p upregulation in GC cells was responsible for the observed SRF repression of E-cadherin. Endogenous E-cadherin protein levels were comparatively high in GC9811 cells, and transfection with either SRF or miR-199a-5p effectively inhibited E-cadherin expression (Figures $6 \mathrm{~d}$ and f). Importantly, miR-199a-5p inhibition abrogated the E-cadherin repression imposed by ectopic SRF expression (Figure 6h), indicating that miR$199 a-5 p$ is a critical mediator of SRF's negative impact on E-cadherin expression.

miR-199a-5p promotes EMT in GC cells and regulates Wnt/ $\beta$-catenin signaling. E-cadherin loss is considered to be a fundamental event in the EMT process. ${ }^{25}$ We therefore questioned whether miR-199a-5p-induced inactivation of $\mathrm{E}$-cadherin leads to EMT in GC metastasis. We observed that ectopic miR-199a-5p expression led to an EMT-like conversion in GES, an immortalized gastric epithelial cell line, and GC9811 cells; these cells became scattered, assumed a spindle-like or star-like morphology (Figure 7a), and displayed a reduction in E-cadherin and an increase in the mesenchymal marker vimentin (Figure $7 b$ ).

As E-cadherin binds to $\beta$-catenin at the cell membrane, preventing its nuclear localization and the transcriptional activation of genes that favor tumor invasion, ${ }^{26}$ we investigated whether miR-199a-5p-mediated E-cadherin suppression affects this pro-metastatic signaling pathway. Immunofluorescence staining demonstrated that $\beta$-catenin was predominantly localized to cell-cell junctions in GES and GC9811 cells. However, miR-199a-5p transfection of GES and GC9811 cells resulted in the translocation of $\beta$-catenin from adherens junctions to the cytoplasm and nucleus (Figure 7c). Together, these results provide evidence that miR-199a-5p promotes EMT and regulates $\mathrm{Wnt} / \beta$-catenin signaling in gastric cells.

\section{Discussion}

Numerous molecules and signaling pathways are involved in tumor metastasis, and these genetic programs are executed 
a

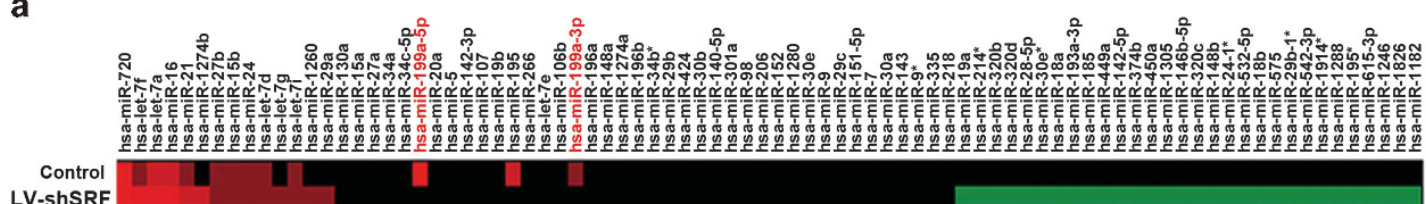
LV-shSRF
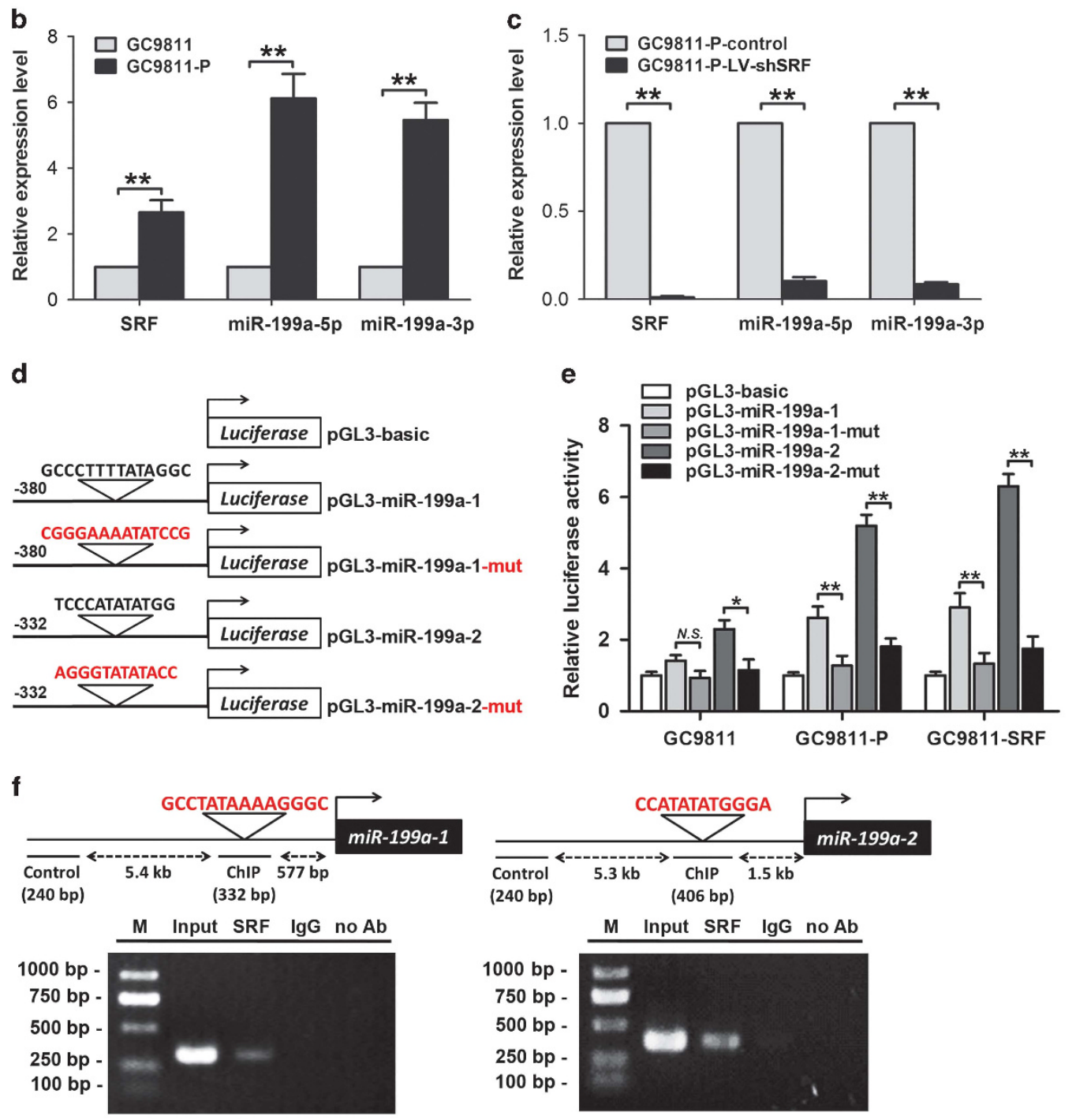

g
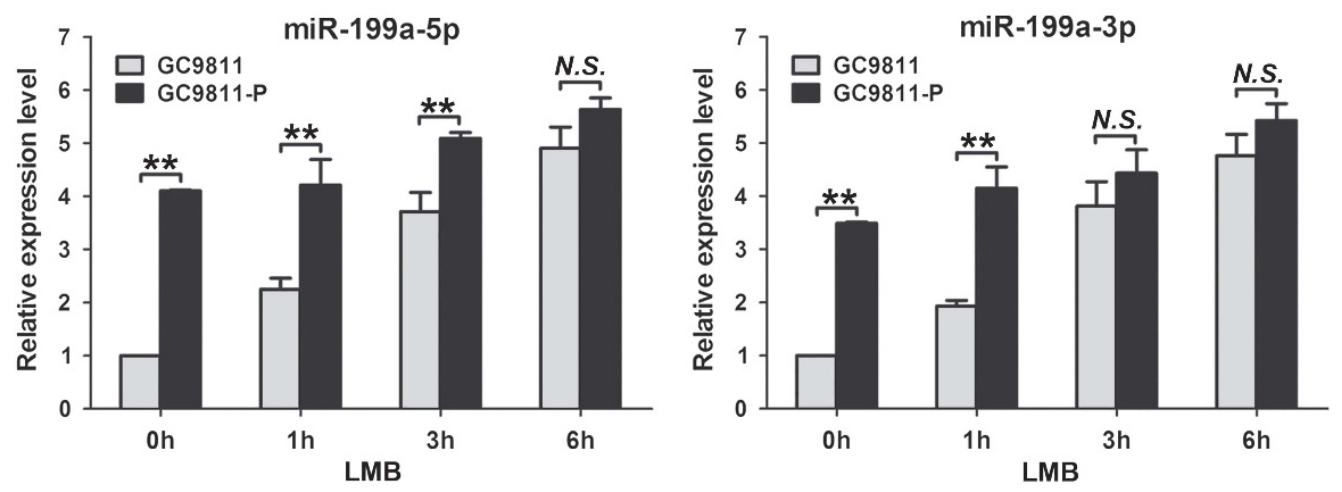
through the co-regulation of functionally related genes by TFs. ${ }^{27,28}$ Systematically analyzing the biochemical activities of TFs provides a way to understand the interactions among TF regulatory control mechanisms that govern gene expression. ${ }^{29-31}$ Using this method, we compared the activities of $\sim 200$ different TFs in a matched pair of primary and metastatic GC cell lines. After expression and functional validation in GC cells and tissues, we recognized the importance of SRF within the regulatory network active in GC cells. In the present study, we investigated SRF's function in GC metastasis and demonstrated that SRF promotes metastasis and EMT through the SRF/miR-199a-5p/E-cadherin pathway.

The metastatic cascade can be conceptually simplified into two major phases: the physical translocation of cancer cells from the primary tumor to a distant tissue and the colonization and outgrowth of translocated cells in a secondary organ. ${ }^{2}$ Here, we investigated the roles of SRF in the multi-step cascade of GC metastatic. (a) For successful translocation in the initial stage, GC cells must acquire the ability to migrate and invade. We found that upregulation of SRF dramatically enhanced the migration and invasion of GC cells. Clinical data confirmed that high-level SRF expression was significantly associated with a more aggressive tumor phenotype and served as a strong and independent predictor of short disease-free survival in GC patients. These findings suggest that SRF has a positive role in the initial step of the GC metastatic cascade. (b) Metastatic colonization is an inefficient, rate-limiting step in the metastatic cascade. In this process, millions of cancer cells are released by the primary tumor and infiltrate the circulatory system each day, but only a small minority of them $(<0.1 \%)$ will develop as distal metastases. ${ }^{32}$ We evaluated the influence of SRF in this stage using a tail vein injection model, which is theoretically analogous to the colonization and outgrowth phases of the tumor metastatic cascade. Our results showed that SRF overexpression increased the number of lung and intrahepatic tumor foci in a GC9811 xenograft model, whereas SRF reduction diminished the colonization of GC9811-P cells. These results suggest that SRF has a pivotal role in multiple rate-limiting steps in the metastatic cascade.

TFs and miRNAs represent the most numerous gene regulatory factors in the genomes of multicellular organisms, and multiple lines of evidence suggest that miRNAs are tightly linked to TFs in gene regulatory networks. This combinatorial regulation by transcriptional and posttranscriptional regulatory mechanisms may provide cells evolutionary advantages. ${ }^{17,33}$ It has been proposed that hundreds to thousands of miRNA genes in mammalian genomes contain at least one
CArG box in their promoter regions, ${ }^{18}$ suggesting that miRNAs may be important targets of SRF. Srivastava and colleagues $^{34}$ showed that SRF regulates the expression of miR-1, which targets Hand2 during cardiogenesis, through the three CArG boxes in its promoter. Liu et al. ${ }^{35}$ also found that SRF could regulate the expression of miR-133a, a critical component in cardiac development and gene expression. In our study, we analyzed changes in miRNA expression by miRNA microarray analysis in GC cells upon SRF silencing and found a positive correlation between SRF and the expression of miR-199a-3p and $-5 p$. Furthermore, we confirmed that SRF could bind to and transactivate both the miR-199a-1 and -2 promoters, thereby upregulating miR-199a expression in GC cells. Of note, Kunkel et al. studied the potential regulatory role of miR-199a-5p in myogenesis and identified SRF as its upstream regulator through computational programs and experimental validation, ${ }^{36}$ suggesting that the SRF/miR-199a-5p regulatory axis might be involved in multiple cellular contexts.

miR-199a has been studied in many tumors owing to its varied and important functions and its identification as both an oncogenic miRNA and tumor suppressor. Cao et al. found that miR-199a/b-3p suppressed HCC growth by inhibiting the PAK4/Raf/MEK/ERK pathway, ${ }^{37}$ and Chan et al. found that miR-199a inhibited proliferation, invasion, and metastasis in testicular cancer. ${ }^{38}$ In contrast, Tavazoie et al. showed that endogenous miR-199a promoted metastatic invasion, angiogenesis, and colonization in melanoma by targeting ApoE and DNAJA4 ${ }^{39}$ In our study, both in vitro and in vivo assays demonstrated that restoration or knockdown of miR-199a-5p, but not miR-199a-3p, affected GC cell migration, invasion, and metastasis, suggesting that it might be the functional effector of SRF in GC metastasis. Moreover, to our knowledge, our study provides the first evidence that miR-199a-5p functions via the regulation of E-cadherin. In accordance with our results, Croce et al. performed a miRNA microarray of 353 gastric samples from two independent subsets of GC patients and recorded miR-199a as one of the three most important miRNAs related to GC progression. ${ }^{40}$ These seemingly divergent functions of miR-199a in various models or systems may be attributed to the different tissues or cellular contexts examined as well as differences in the interaction between miR-199a and its target genes.

Loss of E-cadherin-mediated cell adhesion is a rate-limiting step in the progression from adenoma to carcinoma. Decreased levels of E-cadherin in conjunction with increased levels of mesenchymal-specific proteins,

\footnotetext{
Figure 4 SRF transactivates miR-199a-5p and -3p expression in GC cells. (a) Heatmap showing miRNAs differentially expressed upon SRF silencing in GC9811-P cells. The scale bar shows color-coded differential expression from the mean. (b) Endogenous SRF, miR-199a-5p, and miR-199a-3p were detected in GC9811 and GC9811-P cells by real-time PCR. Values were normalized to GAPDH mRNA levels in the same sample. (c) Expression of SRF, miR-199a-5p, and miR-199a-3p after transfection of GC9811-P cells with LV-shSRF. (d) Schematic of human wild-type or mutant miR-199a-1 and miR-199a-2 promoter constructs fused to a luciferase reporter gene. (e) Luciferase activity in extracts of GC9811, GC9811-SRF, and GC9811-P cells transiently transfected with the wild-type or mutant miR-199a-1 and miR-199a-2 luciferase reporter constructs. Luciferase values are normalized to the empty vector control. (f) Schematic structure of the miR-199a-1 or miR-199a-2 upstream promoter containing a single SRF-binding site. ChIP analyses revealed that SRF binds to the upstream CArG boxes in GC9811-P cells. (g) Real-time PCR analysis of miR-199a-5p and miR-199a-3p expression in GC9811 and GC9811-P cells following treatment with LMB for $0,1,3$, and $6 \mathrm{~h}$. ${ }^{*} P$-value $<0.05$; ${ }^{* *} P$-value $<0.01 ;$ NS, not significant
} 
a

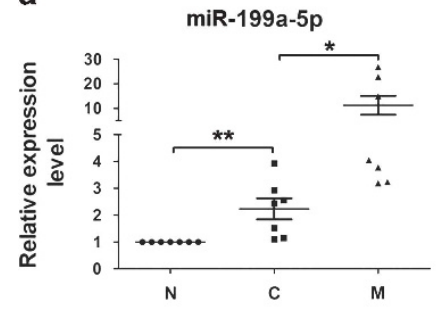

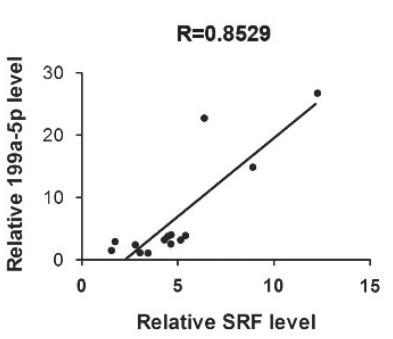

Relative SRF level b

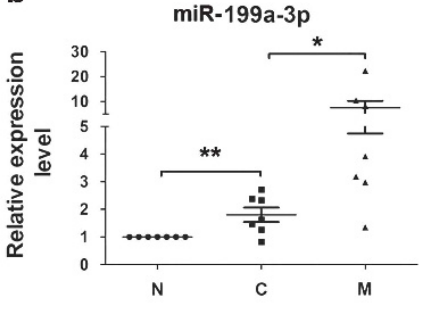

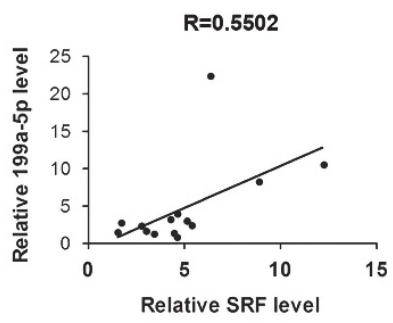

\section{GC9811}

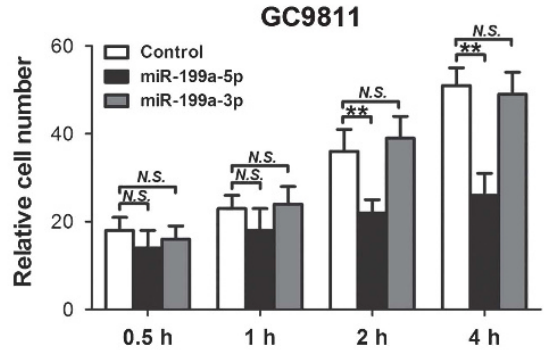

f

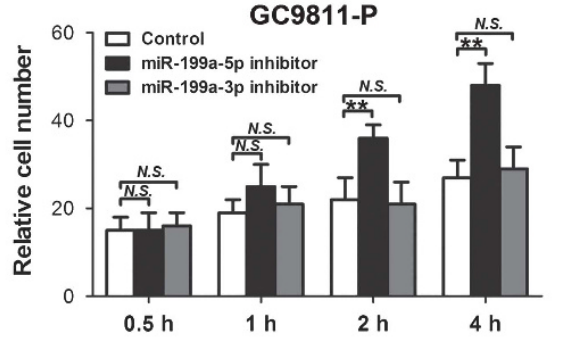

f

g
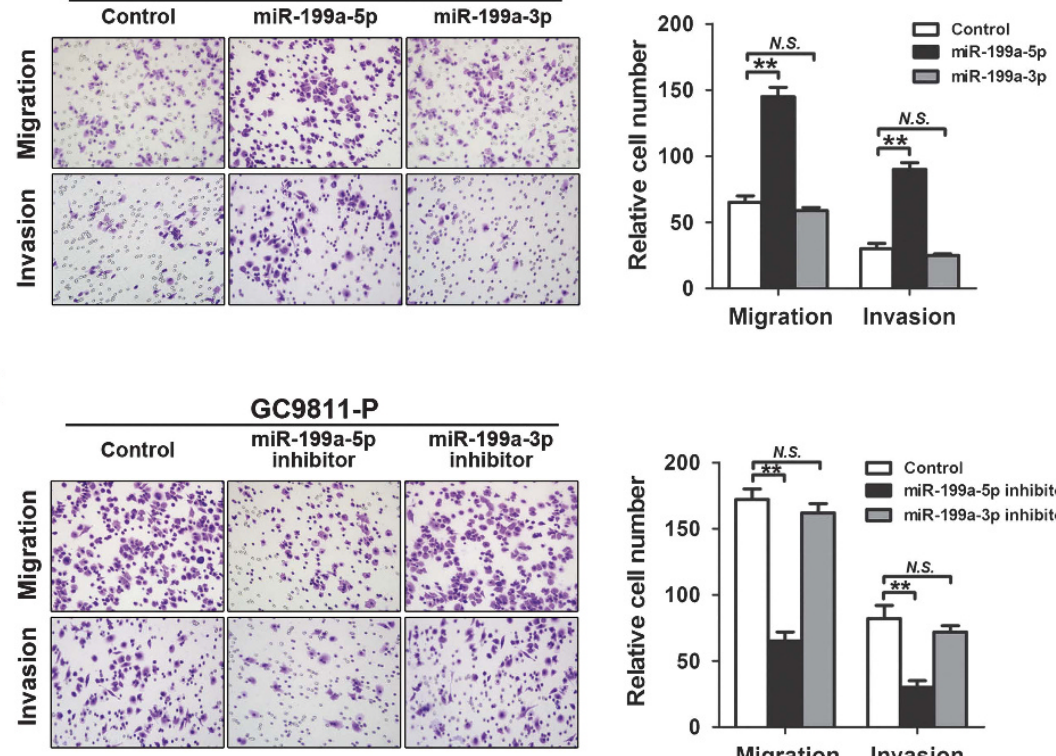

d
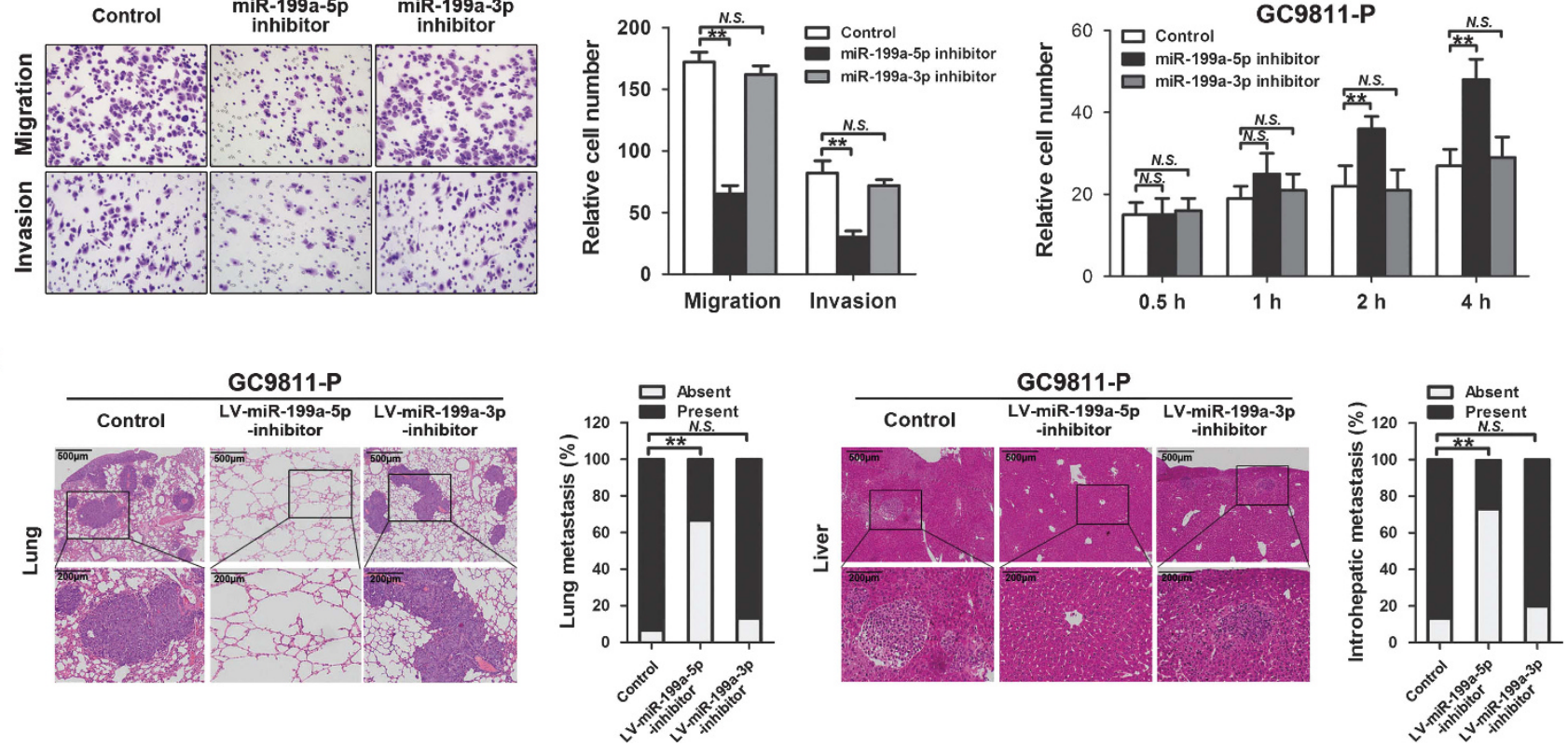

Figure 5 miR-199a-5p inhibits GC cell adhesion and promotes migration, invasion, and metastasis in vitro and in vivo. (a) Relative expression levels of miR-199a-5p and (b) miR-199a-3p in adjacent non-tumor gastric tissues (N), primary GC tissues (C), and lymph node metastatic tissues (M) from seven GC patients. A statistically significant positive correlation was observed between miR-199a-5p/-3p and SRF mRNA levels in GC samples. (c) Transwell migration and invasion assays using GC9811 cells transfected with the miR-199a-5p mimic (miR-199a-5p), the miR-199a-3p mimic (miR-199a-3p), or the control mimic (control) and (d) GC9811-P cells transfected with the miR-199a-5p inhibitor, the miR-199a-3p inhibitor, or the control inhibitor (control). Representative images are shown on the left, and quantification of 10 randomly selected fields is shown on the right. Magnification $=\times 200$. (e) Cell adhesion assays for GC9811 cells transfected with miR-199a-5p, miR-199a-3p, or the control and (f) GC9811-P cells transfected with the miR-199a-5p inhibitor, the miR-199a-3p inhibitor, or the control. Bar graphs represent the average numbers of adhesive cells on the Matrigel after 0.5, 1, 2, and $4 \mathrm{~h}$. (g) Representative H\&E-stained intrahepatic and pulmonary metastases of GC9811-P cells infected with the lentiviral miR-199a-5p inhibitor (LV-miR-199a-5pinhibitor), the miR-199a-3p inhibitor (LV-miR-199a-3p-inhibitor), or the control. Bar graphs represent the percentage of mice with or without metastatic nodules in the liver or the lungs. ${ }^{*} P$-value $<0.05 ;{ }^{* *} P$-value $<0.01$; NS, not significant

such as vimentin, can be used as markers to show that an epithelial cell has undergone EMT. ${ }^{5,25}$ Recent studies have reported that SRF can affect the EMT process, and an inverse correlation between SRF and E-cadherin levels has been observed in both HCC and CRC. ${ }^{11,12}$ We thus performed ChIP experiments to further elucidate the underlying relationship between these genes. Our findings excluded the possibility that SRF inhibits E-cadherin expression through direct transcriptional repression. As miRNAs have been reported as critical regulators of EMT, 
a

Predicted duplex between human CDH1 3'UTR and has-miR-199a-5p

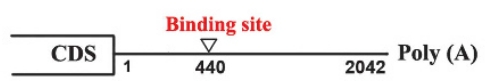

wt 3'-UTR 5'-CUUCUGCUCAUUACUACACUGGU-3'

| | | || || mt 3'-UTR 5'-CUUCUGCUCAUUACUUGUGACCU-3'

C

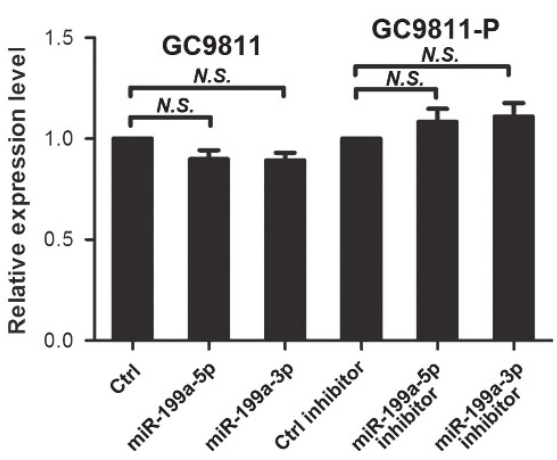

b

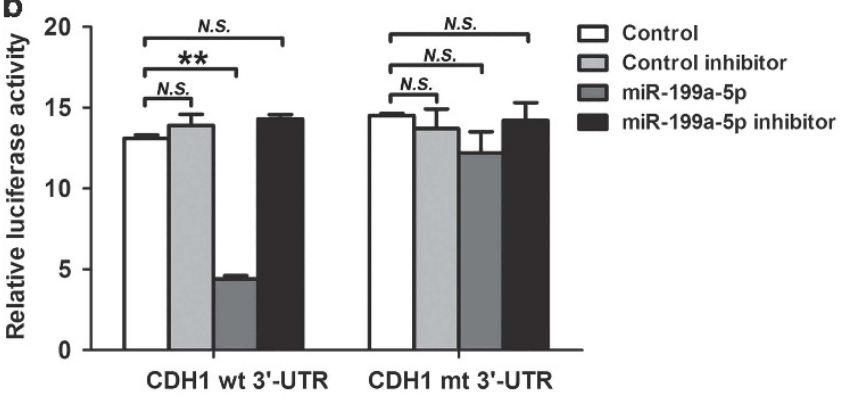

d

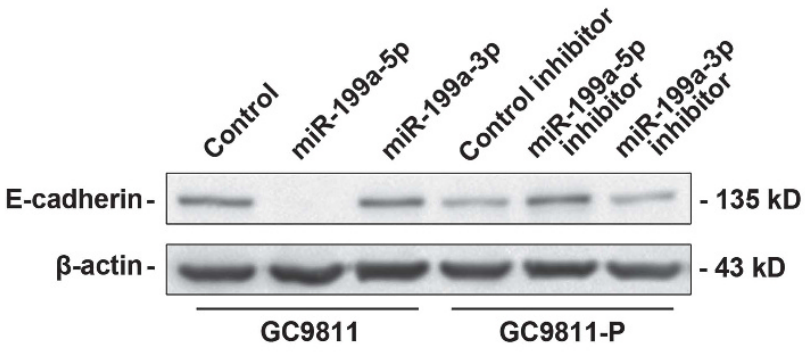

e

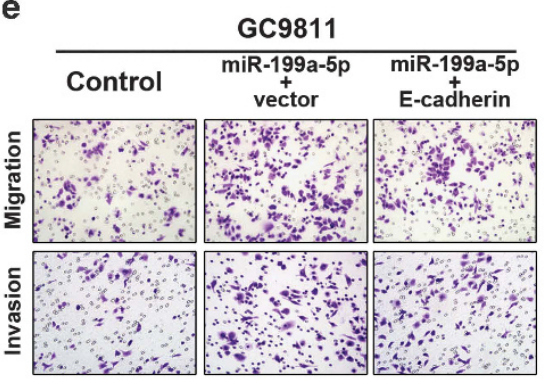

g
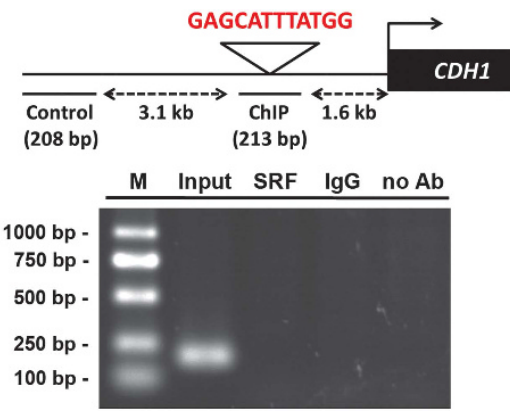

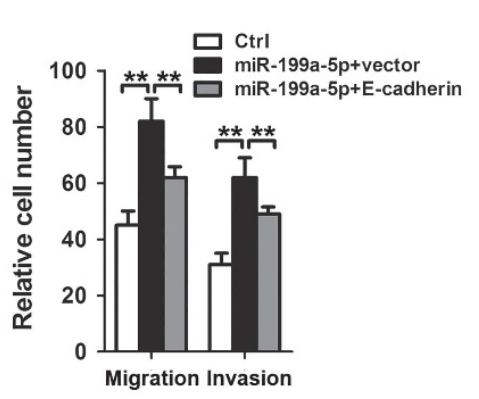

f

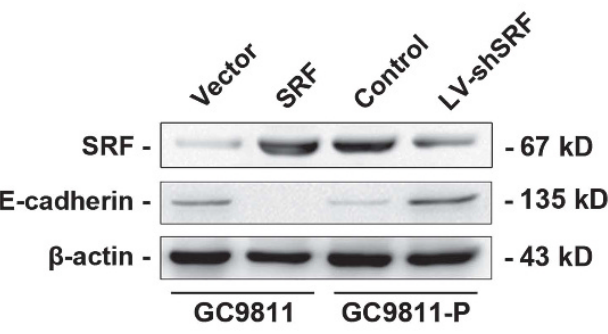

h

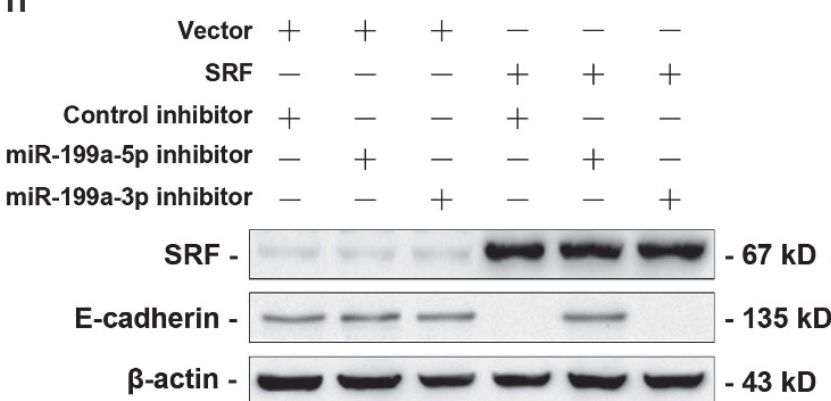

Figure 6 miR-199a-5p directly targets CDH1 in GC cells. (a) Diagram of 3'-UTR luciferase reporter constructs containing the wild-type or mutant miR-199a-5p-binding site. (b) Wild-type or mutant reporter plasmids were co-transfected with miR-199a-5p, miR-199a-5p inhibitor, or control in GC9811 cells. (c) CDH1 expression in GC9811 cells and GC9811-P cells was analyzed by real-time PCR. GAPDH was used as an internal control. (d) E-cadherin protein levels were analyzed by western blot. $\beta$-actin was used as an internal control. (e) Transwell migration and invasion assays for GC9811 cells transfected with miR-199a-5p and transduced with either the CDH1 plasmid (CDH1) or the control plasmid vector (vector). (f) SRF and E-cadherin protein levels were analyzed by western blot. (g) Schematic structure of the CDH1 upstream promoter, which contains a single SRF-binding site. ChIP analysis revealed that SRF could not bind to the upstream CArG box in the CDH1 promoter region in GC9811-P cells. (h) Western blot analysis of SRF and E-cadherin expression in GC9811 cells infected with SRF or vector and transfected with miR-199a-5p inhibitor, miR-199a-3p inhibitor, or control. ${ }^{* *} P$-value $<0.01$; NS, not significant

we thus proposed that SRF might regulate EMT through the transactivation of miR-199a-5p. The SRF/miR-199a-5p/Ecadherin pathway was further experimentally validated as follows: (a) ectopic expression of miR-199a-5p induced an EMT-like conversion in GES and GC9811 cells, accompanied by a decrease in E-cadherin levels and the 
a
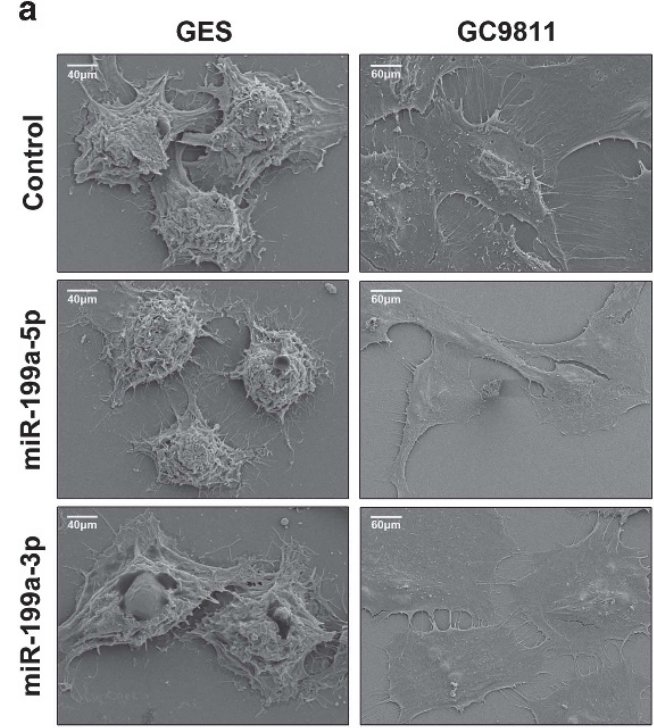

c
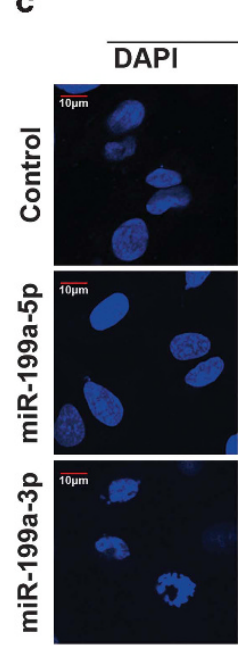

GES
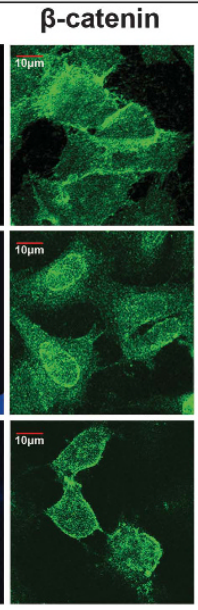

\section{Merge}
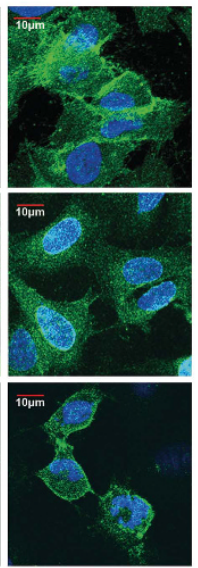

b
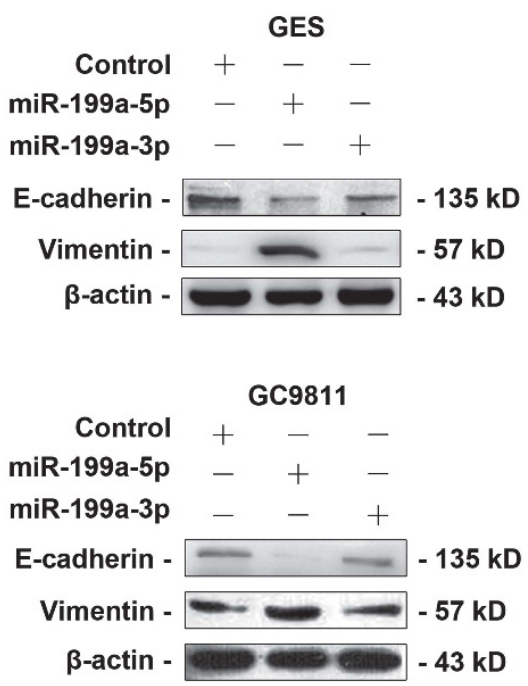

Figure 7 miR-199a-5p promotes EMT in GC cells and regulates Wnt/ $\beta$-catenin signaling. (a) Scanning electron microscope images of GES and GC9811 cells infected with miR-199a-5p, miR-199a-3p, or control for $72 \mathrm{~h}$. Cells were plated in 24-well plates at the same density. (b) Western blot analysis of E-cadherin and vimentin in GES and GC9811 cells transfected with miR-199a-5p, miR-199a-3p, or control. $\beta$-actin was used as a loading control. (c) Immunofluorescence staining of $\beta$-catenin in miR-199a-5p, miR-199a-3p, or control-transfected GES and GC9811 cells, demonstrating the different subcellular localization patterns

translocation of $\beta$-catenin out of cell-cell junctions and (b) inhibition of miR-199a-5p in SRF-expressing GC9811 cells abolished the repression of E-cadherin by SRF. It is worth noting that E-cadherin inactivation has been reported to be accomplished via regulation by various EMT-inducing TFs or miRNAs. ${ }^{5,41}$ In our study, we provide evidence for a novel mode by which E-cadherin and EMT are regulated by the combination of a TF and a miRNA.

In summary, this study investigated the potential role of SRF in GC metastasis and its underlying regulatory mechanisms. Our data suggest that upregulation of SRF promotes GC cell metastasis via the transactivation of miR-199a-5p, which leads to E-cadherin downregulation and EMT induction. This SRF/miR-199a-5p/E-cadherin pathway therefore offers a novel perspective for GC metastasis and may represent an effective therapeutic target for GC treatment.

\section{Materials and Methods}

Cell culture and tissue collection. GC9811, GC9811-P, MKN28-NM, MKN28-M, and GES cells were cultured in RPMI-1640 medium (Thermo Scientific HyClone, Beijing, China). Paired samples of primary GC, adjacent normal tissues, and lymph node metastatic tissues were obtained from patients who had undergone GC surgery at Xijing Hospital, Xi'an, China. All samples were clinically and pathologically shown to be correctly labeled. This study was approved by the hospital's Protection of Human Subjects Committee, and informed consent was obtained from each patient.

High-throughput TF activity profiling. Nuclear extracts were prepared using NE-PER Nuclear and Cytoplasmic Extraction Reagents (Pierce, Rockford, IL, USA) according to the manufacturer's instructions. The TF profiling microarray contained 240 probes, including TF-binding sequences (CapitalBio, Beijing, China). Microarray experiments were performed as previously described. ${ }^{31}$ Array images were acquired using the LuxScan-10KA laser confocal scanner (CapitalBio). The signal intensities of each spot were calculated by subtracting the local background using LuxScan 3.0 software (CapitalBio). 
Protein extraction and western blot. Total protein samples were prepared from fresh-frozen tissues or cultured cells by complete cell lysis (Roche, Mannheim, Germany) with protease and phosphatase. Cytoplasmic and nuclear proteins were isolated using the Nuclear and Cytoplasmic Protein Extraction Kit (Beyotime, Jiangsu, China). Approximately $20-50 \mu \mathrm{g}$ of denatured protein was separated by SDS-PAGE and transferred to nitrocellulose filter membranes (Millipore, Billerica, MA, USA). The antibodies used are listed as follows: anti-SRF (Santa Cruz Biotechnology, Dallas, TX, USA), anti-E-cadherin (Santa Cruz Biotechnology), anti-vimentin (Santa Cruz Biotechnology), anti-histone H3 (Santa Cruz Biotechnology), anti-tubulin (Santa Cruz Biotechnology), and anti- $\beta$-actin (Sigma-Aldrich, St. Louis, MO, USA). The bands were scanned using a ChemiDoc XRS + Imaging System (Bio-Rad, Hercules, CA, USA). The absorbance of the bands was analyzed using image analysis software (ImageJ 1.48) and values were expressed as percentages of SRF/Histone or SRF/Tubulin ratio.

RNA extraction and real-time RT-PCR. Total tissue RNAs were isolated using the RNeasy Kit (Qiagen, Hilden, Germany), and total cellular RNA was extracted using TRIzol reagent (Life Technologies, Carlsbad, CA, USA). The RT-PCR primers for miR-199a-5p, miR-199a-3p, and U6 snRNA were purchased from RiboBio (Guangzhou, China). Reverse transcription PCR was performed using the PrimeScript RT Reagent Kit (TaKaRa, Dalian, China) following the manufacturer's instructions. The PCR primers for SRF and glyceraldehyde 3-phosphate dehydrogenase (GAPDH) were as follows: SRF forward, 5'-GTTCATCGACAACAAGCTGC-3'; SRF reverse, 5'-CTGTCAGCGTGGACA GCTCATAG-3'; GAPDH forward, 5'-ATGTCGTGGA GTCTACTGGC-3'; GAPDH reverse, $5^{\prime}$-TGACCTTGCCCACAGCCTTG- $3^{\prime}$. Real-time PCR was performed using SYBR Premix Ex Taq II (TaKaRa) and a LightCycler 480 system (Roche, Basel, Switzerland). The level of U6 or GAPDH was used as an internal control. The $2^{-\Delta \Delta C T}$ method was used to determine fold changes in the RNA levels of each sample compared with the reference sample.

Tissue microarray and immunohistochemistry. GC tissue microarrays chips (No. CC01-01, CC01-21), including 25 normal gastric tissues, 28 GC tissues, and $48 \mathrm{GC}$ lymph node metastatic tissues, were obtained from Shaanxi Chaoying Biotechnology Company. GC tissue microarray chips with follow-up data (No. HStm-Ade178Sur-01) containing 67 GC tissues paired with matched adjacent normal tissues, 39 gastric malignant tissues, and 5 adjacent tissues were obtained from Shanghai Biochip Company. Immunohistochemistry was conducted as previously described ${ }^{42}$ using an anti-SRF antibody (Santa Cruz Biotechnology). All sections were examined and scored independently by two investigators in a blind manner. Expression levels were visualized and classified based on the percentage of positive cells and the staining intensity.

Immunofluorescence staining. Indirect immunofluorescence staining for SRF and $\beta$-catenin was performed on GC cells and frozen sections of xenografted tumor tissues using anti-SRF (Santa Cruz Biotechnology) and anti- $\beta$-catenin (Cell Signaling, Danvers, MA, USA) antibodies as previously described. ${ }^{43}$

Plasmids and Oligonucleotides transfection. The human SRF expression plasmid was kindly provided by Dr. Eric Olson (University of Texas Southwestern Medical Center). The pcDNA3-CDH1 plasmid was constructed as previously described ${ }^{43}$ by inserting the $\mathrm{CDH} 1 \mathrm{CDNA}$ into the pcDNA3 vector (Life Technologies) at the $\mathrm{Xhol}$ and $\mathrm{Xbal}$ sites. Transfections were performed using Lipofectamine 2000 (Life Technologies) according to the manufacturer's instructions. siRNA, miRNA mimic, and miRNA inhibitor transfection were performed following the same procedure. The sense strand sequences of SRF siRNAs designed to target human cells were as follows: SRF siRNA no. 1, 5'-CCACAACAGACCAGAGAAUTT-3'; SRF SIRNA no. 2, 5'-GU UCCUGACAG CAUCAUCUTT-3'; SRF SiRNA no. 3, 5'-CCCUGUUUCAGCAGUUCAGTT-3'. Successful overexpression and knockdown of SRF were confirmed by western blot (Supplementary Figure S1). The miR-199a-5p mimic (miR-199a-5p), miR-199a-3p mimic (miR-199a-3p), miRNA mimic negative control, miR-199a-5p inhibitor, miR-199a-3p inhibitor, and miRNA inhibitor negative control oligonucleotides were chemically synthesized and purified by RiboBio (Ribobio).

Lentivirus production and transduction. The lentiviral vector containing SRF DNA sequence (LV-SRF), lentiviral vector containing SRF siRNA no. 3 hairpin sequence (LV-shSRF), lentiviral miR-199a-5p inhibitor (LV-miR-199a-5p-inhibitor), lentiviral miR-199a-3p inhibitor (LV-miR-199a-3p-inhibitor), and negative control were constructed by Shanghai GeneChem. Constructs containing the sequences of SRF, SRF shRNA, miR-199a-3p inhibitor, or miR-199a-5p inhibitor sequence plus a 100-bp sequence of upstream and downstream flanking sides were cloned into the pGCSIL-GFP vector. Target cells $\left(1 \times 10^{5}\right)$ were infected with $1 \times 10^{7}$ lentivirus transducing units in the presence of $10 \mu \mathrm{g} / \mathrm{ml}$ polybrene.

Migration and invasion assays. For migration assays, infected or transfected cells were harvested and resuspended in serum-free RPMI-1640 medium, and $1 \times 10^{5}$ cells were placed into Boyden chambers (Corning, Tewksbury, MA, USA) with an $8.0 \mu \mathrm{m}$ pore membrane. For invasion assays, $1 \times 10^{5}$ cells were placed into chambers coated with $150 \mathrm{mg}$ of Matrigel (BD Biosciences, Boston, MA, USA). The chambers were then inserted into the wells of a 24-well plate and incubated for $24 \mathrm{~h}$ in RPMI- 1640 medium with $10 \%$ fetal bovine serum prior to examination. The cells remaining on the upper surface of the membrane were removed, and the cells adhering to the lower surface were fixed, stained in a dye solution containing $0.05 \%$ crystal violet, and counted under a microscope (Olympus, Tokyo, Japan) to determine their relative numbers.

Cell adhesion assays. The ability of GC cells to adhere to Matrigel $(50 \mu \mathrm{g} / \mathrm{ml}$, BD Biosciences) was assessed in 24-well plates as previously described ${ }^{44}$ The plate surface was coated with $0.2 \mathrm{ml}$ of Matrigel and incubated for $2 \mathrm{~h}$, and then the supernatant was removed. A $0.5 \mathrm{ml}$ suspension of tumor cells $\left(1 \times 10^{5} / \mathrm{ml}\right)$ was transferred into the covered wells. After $0.5,1,2$, and $4 \mathrm{~h}$ of incubation at $37^{\circ} \mathrm{C}$, the adhesive cells were stained with crystal violet and evaluated at $\times 200$ magnification in 10 random fields of each well according to the manufacturer's instructions (Life Technologies).

In vivo metastasis assay. For the in vivo metastasis assays, $2 \times 10^{6} \mathrm{GC}$ cells infected with LV-SRF, LV-shSRF, LV-miR-199a-5p-inhibitor, LV-miR-199a$3 p$-inhibitor, or negative control were suspended in $200 \mathrm{ml}$ of phosphate-buffered saline and injected into the tail vein of nude mice (15 in each group, female nu/nu, purchased from Shanghai Laboratory Animal Center of China). The mice were killed 4 weeks later, and the lungs and tumor tissues derived from various organs were dissected and examined. All animal studies complied with the animal use guidelines of Fourth Military Medical University, and the protocols were approved by the Fourth Military Medical University Animal Care Committee.

miRNA expression profiling. RNA was extracted using the RNeasy mini column (Qiagen). The miRNA expression profiles of GC9811-P control and GC9811-P-shSRF cells were assessed using the Affymetrix Chip Human Array (Affymetrix, Santa Clara, CA, USA), as previously described. ${ }^{45}$

Chromatin immunoprecipitation. ChIP assays were carried out as previously described ${ }^{46}$ using an anti-SRF antibody (Santa Cruz Biotechnology). Ten percent of chromatin prior to immunoprecipitation was used as input controls and nonspecific antibody (rabbit anti-lgG; BD Biosciences) served as negative controls. The precipitated DNAs were subjected to PCR in attempt to amplify the SRF-binding sites using primers specific for miR-199a-1 (forward, 5'-GGCTCTCTTCTCGGCCGCTG-3'; Reverse, $\quad$ 5'-CGCTGATGCCTCTAAGG CGC-3') and miR-199a-2 (forward, 5'-GTCTTGACGTGGCACATTTGC-3'; Reverse, $5^{\prime}$-CATTTTATGCAC AGACCCATGTC- $\left.3^{\prime}\right)$. The amplified fragments were then resolved electrophoretically on a $2 \%(\mathrm{w} / \mathrm{v})$ agarose gel and verified by DNA sequencing.

Luciferase reporter assays. To construct promoter reporter vectors, the wild-type miR-199a-1 or miR-199a-2 promoter containing putative binding site for SRF was PCR-amplified using genomic DNA from GC9811 cells as a template. The SRF-binding sites in miR-199a-1 and -2 were subjected to site-directed mutagenesis to create the corresponding mutant constructs. Both wild-type and mutant promoter were inserted upstream of the Firefly luciferase reporter in the pGL3-basic vector. To construct miRNA $3^{\prime}$-UTR luciferase reporter vectors, the wild-type $3^{\prime}$-UTR of CDH1, containing putative binding sites for miR-199a-5p, was PCR-amplified using genomic DNA from GC9811 cells as a template. The corresponding mutant constructs were created by mutating the seed regions of the miR-199a-5p-binding sites. Both wild-type and mutant $3^{\prime}$-UTRs were cloned downstream of the luciferase gene in the psiCHECK-2 luciferase vector. All the above constructs were verified by sequencing. GC9811, GC9811 stably expressing SRF (GC9811-SRF), or GC9811-P cells 
were transfected with the appropriate plasmids in 24-well plates. Cells were harvested and lysed for luciferase assays $48 \mathrm{~h}$ after transfection. Luciferase assays were performed using a Dual-Luciferase Reporter Assay System (Promega, Madison, WI, USA) according to the manufacturer's protocol. Firefly luciferase activity normalized to Renilla luciferase was used as an internal control. The transfection experiments were performed in triplicate for each plasmid construct.

Morphological analysis by scanning electron microscopy. Scanning electron microscopy imaging was conducted as previously described. ${ }^{47}$ Cells were cultured on glass cover slips and fixed with $2.5 \%$ glutaraldehyde in $0.1 \mathrm{M}$ sodium cacodylate buffer $(\mathrm{pH} 7.2)$ for $30 \mathrm{~min}$. The samples were washed with cacodylate buffer, dehydrated through a graded series of ethanol, and dried with hexamethyldisilazane. The samples were then mounted on aluminum stubs and coated with gold/palladium $(10 \mathrm{~nm})$. The cells were examined under a scanning electron microscope (Hitachi 3400N, Tokyo, Japan) at $3.00 \mathrm{kV}$.

Statistical analysis. The SPSS 12.0 program (SPSS, Chicago, IL, USA) was used for statistical analysis. Experimental data are expressed as the means \pm S.E. Differences between means were assessed using Student's $t$-test, ANOVA, or $\chi^{2}$-tests. Significant differences were determined by ANOVA followed by post hoc comparisons with Fisher's protected least significant difference test. Data were considered statistically significant at $P<0.05$.

\section{Conflict of Interest}

The authors declare no conflict of interest.

Acknowledgements. We acknowledge Dr. Eric Olson from University of Texas Southwestern Medical Center for providing the full-length human SRF plasmid. This work was supported by the National Key and Basic Research Development Program of China (973 Program, no. 2010CB529300, 05, 02, 06) and the National Natural Science Foundation of China (no. 81030044, 81120108005, 81272652, 81225003, 81227901, 81270849, 81270445, 81201929, 81201632, 81272347). We also acknowledge the financial support from China Scholarship Council (no. 201306590015).

1. Jemal A, Bray F, Center MM, Ferlay J, Ward E, Forman D. Global cancer statistics. CA Cancer J Clin 2011; 61: 69-90.

2. Chaffer CL, Weinberg RA. A perspective on cancer cell metastasis. Science 2011; 331: 1559-1564.

3. Shah MA, Ajani JA. Gastric cancer-an enigmatic and heterogeneous disease. JAMA 2010; 303: $1753-1754$.

4. Fidler IJ. The pathogenesis of cancer metastasis: the 'seed and soil' hypothesis revisited. Nat Rev Cancer 2003; 3: 453-458.

5. Thiery JP, Acloque H, Huang RY, Nieto MA. Epithelial-mesenchymal transitions in development and disease. Cell 2009; 139: 871-890.

6. Medjkane S, Perez-Sanchez C, Gaggioli C, Sahai E, Treisman R. Myocardin-related transcription factors and SRF are required for cytoskeletal dynamics and experimental metastasis. Nat Cell Biol 2009; 11: 257-268.

7. Ozanne BW, Spence HJ, McGarry LC, Hennigan RF. Transcription factors control invasion: AP-1 the first among equals. Oncogene 2007; 26: 1-10.

8. Wu Y, Zhou BP. TNF-alpha/NF-kappaB/Snail pathway in cancer cell migration and invasion. Br J Cancer 2010; 102: 639-644.

9. Shore $P$, Sharrocks AD. The MADS-box family of transcription factors. Eur J Biochem 1995; 229: 1-13.

10. Miano JM. Role of serum response factor in the pathogenesis of disease. Lab Invest 2010; 90: 1274-1284.

11. Park MY, Kim KR, Park HS, Park BH, Choi HN, Jang KY et al. Expression of the serum response factor in hepatocellular carcinoma: implications for epithelial-mesenchymal transition. Int J Oncol 2007; 31: 1309-1315.

12. Choi HN, Kim KR, Lee JH, Park HS, Jang KY, Chung MJ et al. Serum response factor enhances liver metastasis of colorectal carcinoma via alteration of the E-cadherin/betacatenin complex. Oncol Rep 2009; 21: 57-63.

13. Kwon CY, Kim KR, Choi HN, Chung MJ, Noh SJ, Kim DG et al. The role of serum response factor in hepatocellular carcinoma: implications for disease progression. Int J Oncol 2010; 37: 837-844.

14. Kim KR, Bae JS, Choi HN, Park HS, Jang KY, Chung MJ et al. The role of serum response factor in hepatocellular carcinoma: an association with matrix metalloproteinase. Oncol Rep 2011; 26: 1567-1572.
15. Bartel DP. MicroRNAs: genomics, biogenesis, mechanism, and function. Cell 2004; 116: 281-297.

16. White NM, Fatoohi E, Metias M, Jung K, Stephan C, Yousef GM. Metastamirs: a stepping stone towards improved cancer management. Nat Rev Clin Oncol 2011; 8 $75-84$.

17. Hobert $\mathrm{O}$. Gene regulation by transcription factors and microRNAs. Science $2008 ; 319$ : 1785-1786.

18. Niu Z, Li A, Zhang SX, Schwartz RJ. Serum response factor micromanaging cardiogenesis Curr Opin Cell Biol 2007; 19: 618-627.

19. Park C, Hennig GW, Sanders KM, Cho JH, Hatton WJ, Redelman D et al. Serum response factor-dependent MicroRNAs regulate gastrointestinal smooth muscle cell phenotypes. Gastroenterology 2011; 141: 164-175.

20. Jean D, Bar-Eli M. Regulation of tumor growth and metastasis of human melanoma by the CREB transcription factor family. Mol Cell Biochem 2000; 212: 19-28.

21. Miano JM, Long $X$, Fujiwara K. Serum response factor: master regulator of the actin cytoskeleton and contractile apparatus. Am J Physiol Cell Physiol 2007; 292 C70-C81.

22. Ziegler EC, Ghosh S. Regulating inducible transcription through controlled localization. Sci STKE 2005; 2005: e6.

23. Valastyan S, Weinberg RA. Tumor metastasis: molecular insights and evolving paradigms. Cell 2011; 147: 275-292.

24. Onder TT, Gupta PB, Mani SA, Yang J, Lander ES, Weinberg RA. Loss of E-cadherin promotes metastasis via multiple downstream transcriptional pathways. Cancer Res 2008; 68: 3645-3654.

25. Perl AK, Wilgenbus $P$, Dahl U, Semb H, Christofori G. A causal role for E-cadherin in the transition from adenoma to carcinoma. Nature 1998; 392: 190-193.

26. Schmalhofer $\mathrm{O}$, Brabletz S, Brabletz T. E-cadherin beta-catenin, and ZEB1 in malignant progression of cancer. Cancer Metastasis Rev 2009; 28: 151-166.

27. Chiang AC, Massague J. Molecular basis of metastasis. N Engl J Med 2008; 359 : 2814-2823.

28. Weber GF. Molecular mechanisms of metastasis. Cancer Lett 2008; 270: $181-190$.

29. Zhao Y, Shao W, Wei H, Qiao J, Lu Y, Sun $Y$ et al. Development of a novel oligonucleotide array-based transcription factor assay platform for genome-wide active transcription factor profiling in Saccharomyces cerevisiae. J Proteome Res 2008; 7: 1315-1325.

30. Qiao JY, Shao W, Wei HJ, Sun YM, Zhao YC, Xing WL et al. Novel high-throughput profiling of human transcription factors and its use for systematic pathway mapping. J Proteome Res 2008; 7: 2769-2779.

31. Sun YM, Zhang Y, Zeng LQ, Wu JP, Wei L, Ren AH et al. Broad profiling of DNA-binding transcription factor activities improves regulatory network construction in adult mouse tissues. J Proteome Res 2008; 7: 4455-4464.

32. Gupta GP, Massague J. Cancer metastasis: building a framework. Cell 2006; 127 679-695.

33. Zhou Y, Ferguson J, Chang JT, Kluger Y. Inter- and intra-combinatorial regulation by transcription factors and microRNAs. BMC Genomics 2007; 8: 396.

34. Zhao $Y$, Samal E, Srivastava D. Serum response factor regulates a muscle-specific microRNA that targets Hand2 during cardiogenesis. Nature 2005; 436: 214-220.

35. Liu N, Bezprozvannaya S, Williams AH, Qi X, Richardson JA, Bassel-Duby R et al. microRNA-133a regulates cardiomyocyte proliferation and suppresses smooth muscle gene expression in the heart. Genes Dev 2008; 22: 3242-3254.

36. Alexander MS, Kawahara G, Motohashi N, Casar JC, Eisenberg I, Myers JA et al. MicroRNA-199a is induced in dystrophic muscle and affects WNT signaling, cell proliferation, and myogenic differentiation. Cell Death Differ 2013; 20: 1194-1208.

37. Hou J, Lin L, Zhou W, Wang Z, Ding G, Dong Q et al. Identification of miRNomes in human liver and hepatocellular carcinoma reveals miR-199a/b-3p as therapeutic target for hepatocellular carcinoma. Cancer Cell 2011; 19: 232-243.

38. Cheung HH, Davis AJ, Lee TL, Pang AL, Nagrani S, Rennert OM et al. Methylation of an intronic region regulates miR-199a in testicular tumor malignancy. Oncogene 2011; 30: 3404-3415.

39. Pencheva N, Tran H, Buss C, Huh D, Drobnjak M, Busam K et al. Convergent multi-miRNA targeting of ApoE drives LRP1/LRP8-dependent melanoma metastasis and angiogenesis. Cell 2012; 151: 1068-1082.

40. Ueda T, Volinia S, Okumura H, Shimizu M, Taccioli C, Rossi S et al. Relation between microRNA expression and progression and prognosis of gastric cancer: a microRNA expression analysis. Lancet Oncol 2010; 11: 136-146.

41. Zhao X, Lu Y, Nie Y, Fan D. MicroRNAs as critical regulators involved in regulating epithelial-mesenchymal transition. Curr Cancer Drug Targets 2013; 13 935-944.

42. Li T, Lu YY, Zhao XD, Guo HQ, Liu CH, Li H et al. MicroRNA-296-5p increases proliferation in gastric cancer through repression of Caudal-related homeobox 1 Oncogene 2014; 33: 783-793.

43. Zhao X, Dou W, He L, Liang S, Tie J, Liu C et al. MicroRNA-7 functions as an antimetastatic microRNA in gastric cancer by targeting insulin-like growth factor-1 receptor Oncogene 2013; 32: 1363-1372. 
44. Thamilselvan V, Basson MD. Pressure activates colon cancer cell adhesion by inside-out focal adhesion complex and actin cytoskeletal signaling. Gastroenterology 2004; 126: 8-18.

45. Suzuki HI, Yamagata K, Sugimoto K, Iwamoto T, Kato S, Miyazono K. Modulation of microRNA processing by p53. Nature 2009; 460: 529-533.

46. Vian L, Di Carlo M, Pelosi E, Fazi F, Santoro S, Cerio AM et al. Transcriptional fine-tuning of microRNA-223 levels directs lineage choice of human hematopoietic progenitors. Cell Death Differ 2014; 21: 290-301.

47. Takkunen M, Hukkanen M, Liljestrom M, Grenman R, Virtanen I. Podosome-like structures of non-invasive carcinoma cells are replaced in epithelial-mesenchymal transition by actin comet-embedded invadopodia. J Cell Mol Med 2010; 14: 1569-1593. (c) (1) () $\odot$ This work is licensed under a Creative Commons Attribution-NonCommercial-NoDerivs 3.0 Unported License. The images or other third party material in this article are included in the article's Creative Commons license, unless indicated otherwise in the credit line; if the material is not included under the Creative Commons license, users will need to obtain permission from the license holder to reproduce the material. To view a copy of this license, visit http://creativecommons.org/licenses/by-nc-nd/3.0/

Supplementary Information accompanies this paper on Cell Death and Differentiation website (http://www.nature.com/cdd) 